\title{
Green Nanotechnology of MGF-AuNPs for Immunomodulatory Intervention in Prostate Cancer Therapy
}

\author{
Menka Khoobchandani \\ University of Missouri
}

\section{Aslam Khan}

University of Missouri

Kavita Katti

University of Missouri

Velaphi Thipe

Instituto de Pesquisas Energéticas e Nucleares

Amal Al-Yasiri

University of Missouri

\section{Darsha MohanDoss}

Dhanvantari Nano

\section{Michael Nicholl}

South Texas Veterans Health Care System

Ademar Lugão

Instituto de Pesquisas Energéticas e Nucleares

Kattesh Katti ( $\sim$ KattiK@health.missouri.edu )

University of Missouri

\section{Research Article}

Keywords: Mangiferin, gold nanoparticles, NF-kB, laminin receptor, prostate tumor, macrophages

Posted Date: February 5th, 2021

DOl: https://doi.org/10.21203/rs.3.rs-135140/v1

License: (c) (i) This work is licensed under a Creative Commons Attribution 4.0 International License. Read Full License

Version of Record: A version of this preprint was published at Scientific Reports on August 18th, 2021. See the published version at https://doi.org/10.1038/s41598-021-96224-8. 



\title{
Green Nanotechnology of MGF-AuNPs for Immunomodulatory Intervention in Prostate Cancer Therapy
}

\author{
Menka Khoobchandani ${ }^{1}$, Aslam Khan ${ }^{2}$, Kavita K Katti ${ }^{1}$, Velaphi C Thipe ${ }^{3}$, Amal. Y. Al-Yasiri ${ }^{4}$, \\ Darsha K D MohanDoss ${ }^{5}$, Michael Nicholl ${ }^{6}$, Ademar B. Lugão ${ }^{3}$, Kattesh V Katti ${ }^{1,7,8 *}$ \\ ${ }^{1}$ Department of Radiology, Institute of Green Nanotechnology, University of Missouri, \\ Columbia, MO, 65212, USA \\ ${ }^{2}$ Department of Biochemistry, University of Missouri, Columbia, MO, 65212, USA \\ ${ }^{3}$ Laboratório de Ecotoxicologia - Centro de Química e Meio Ambiente - Instituto de Pesquisas \\ Energéticas e Nucleares (IPEN) - Comissão Nacional de Energia Nuclear- IPEN/CNEN-SP, \\ Butantã, São Paulo - SP, Brasil \\ ${ }^{4}$ Nuclear Science and Engineering Institute (NSEI), University of Missouri, Columbia, MO \\ 65211, USA \\ ${ }^{5}$ Dhanvantari Nano Ayushadi Pvt Ltd, No. 8/34, Neelakanta Mehta Street, T. Nagar, Chennai - \\ 600017-India \\ ${ }^{6}$ South Texas VA Health Care San Antonio, Texas, USA \\ ${ }^{7}$ Department of Physics, ${ }^{8}$ University of Missouri Research Reactor (MURR), University of \\ Missouri, Columbia, MO, 65212, USA
}

*Correspondence: E mail: KattiK@,health.missouri.edu

\begin{abstract}
Men with castration-resistant prostate cancer (CRPC) face poor prognosis and increased risk of treatment-incurred adverse effects resulting in one of the highest mortalities among patient population globally. Immune cells act as double-edged sword depending on the tumor microenvironment, which leads to increased infiltration of pro-tumor (M2) macrophages. Development of new immunomodulatory therapeutic agents capable of targeting the tumor microenvironment, and hence orchestrating the differentiation of pro-tumor M2 macrophages to anti-tumor M1, would substantially improve treatment outcomes of CRPC patients. We report, herein, Mangiferin functionalized gold nanoparticles (MGF-AuNPs) and its immunomodulatory characteristics in treating prostate cancer. We provide evidence of immunomodulatory intervention of MGF-AuNPs in prostate cancers through observations of enhanced levels of antitumor cytokines (IL-12 and TNF- $\alpha$ ) with concomitant reductions in the levels of pro-tumor cytokines (IL-10 and IL-6). In the treated groups, IL-12 was elevated to ten-fold while TNF- $\alpha$ was elevated to about fifty-fold; while IL-10 and IL-6 were reduced by two-fold. Ability of MGF-AuNPs to target splenic macrophages is invoked via targeting of NF-kB signaling pathway. Finally, therapeutic efficacy of MGF-AuNPs, in treating prostate cancer in vivo in tumor bearing mice, is described taking into consideration various immunomodulatory interventions triggered by this green nanotechnology-based nanomedicine agent.
\end{abstract}

Keywords. Mangiferin, gold nanoparticles, NF- $\mathrm{BB}$, laminin receptor, prostate tumor, macrophages. 


\section{Introduction}

The latest epidemiological investigation (spanning January 1, 2008-March 31, 2018) of patients with castration-resistant prostate cancer (mCRPC) has concluded high mortality suggesting a significant unmet clinical need in prolonging life span of human population inflicted with this deadly disease globally. ${ }^{1-4}$ There is an emerging consensus that current therapies are poorly effective for patients with castration-resistant prostate cancer (CRPC), where the disease manifests from asymptomatic or minimally symptomatic, non-metastatic disease to symptomatic or highly metastatic condition, depending on the time of diagnosis with significant interpatient variation. ${ }^{5-7}$ The United States Food and Drug Administration (FDA) has approved several chemotherapeutic agents including docetaxel, cabazitaxel, abiraterone, and enzalutamide for treating such patients. Drug resistance attributable to modulation of myeloid-derived suppressor cells (MDSCs) is seen in a significant proportion of CRPC patients. Myeloid-derived suppressor cells (MDSCs) induce an immune suppressive microenvironment and promote the M2-polarized tumor associated macrophages (TAMs). These macrophages present remarkable ability to suppress T-cell responses thus supporting angiogenesis and metastasis of CRPC. Macrophages, the myeloid derived, immune cells of the innate immune system, manifest two states of polarization (M1 and M2) that develop in direct response to different stimuli. The polarization and differentiation of macrophages into the cancer-inhibiting M1 and cancer-promoting M2 phenotypes represent the two states of macrophages in the tumor microenvironment. ${ }^{8-10}$

Numerous studies have also shown that tissue and serum exosomes from prostate cancer patients induced higher levels of macrophage polarization into an alternatively activated M2 (pro-tumor) phenotype. ${ }^{11-13}$ The interaction of polarized macrophages with cancer cells plays a crucial role in a variety of cancers including prostate cancers. ${ }^{9,14,15}$ Several investigations have provided important insights on the role of the polarization of macrophages from M1 into M2 phenotypes and how this macrophage axis is directly involved in the prostate cancer initiation, progression, and metastasis. ${ }^{16-18}$ An additional contributing factor for enhanced levels of protumor M2 phenotypes in a vast majority of prostate and most solid tumors is attributed to elevated NF- $\mathrm{B}$ signaling, upregulated by the release of cytokines by M2 macrophages, in the tumor microenvironment. ${ }^{19,20}$ Compelling evidence shows that chemotherapeutic and radiation treatments of solid cancers in general, and prostate tumors in particular, activates NF- $\mathrm{B}$, a key transcription factor that plays a critical role in the development and progression of cancer and consequently aiding chemo and multi therapy drug resistance. Upregulated NF- $\kappa \mathrm{B}$ activity can activate pro-survival pathways, including BCL-2. Therefore, cancer treatment emphasizing personalized therapy, through immune boosting precision medicine, capable of targeting M2 macrophages is distinguished from a plethora of "common denominator" treatment approaches in current use. ${ }^{21-23}$

In the context of developing novel therapies for treating drug-resistant cancers such as castration-resistant prostate cancer (CRPC), high antioxidant capacity and immunomodulatory phytochemicals are gaining considerable scientific and clinical interests. ${ }^{24,25}$ Anti-neoplastic activity of phytochemicals mainly depends on their multi-target mechanism of action, including their ability to modulate the host immune response to cancer, reducing inflammatory microenvironment and enhancing lymphocyte oncosurveillance. ${ }^{26-28}$ Numerous therapeutic effects of various phytochemicals are believed to be based on mechanisms of modulation of innate immunity more specifically macrophage function. ${ }^{29,30}$ Since carcinogenesis is multifactorial activity involving several signaling pathways, multi targeted phytochemicals therefore represent a promising therapeutic domain in oncology. ${ }^{31,32}$ However, one of the major 
challenges, which continue to impede the application of phytochemicals, in cancer treatment is associated with achieving adequate bioavailability at tolerable doses. This is a vexing problem in translating promising findings from cell culture and animal models into clinically efficacious phytochemical-based drugs.

Nanotechnology offers practical and scientifically most effective means to create multitudes of signatures of phytochemicals on individual nanoparticles - thus enhancing bioavailability to achieve optimum therapeutic payloads at the tumor site. ${ }^{33-35}$ Over the last two decades, we have successfully demonstrated that large surface area of gold nanoparticles can be embedded with a plethora of phytochemicals to create biocompatible cancer therapeutic nanomedicine agents. ${ }^{33-51}$ Our extensive results, using tumor bearing rodents as well as in tumor bearing dogs (where the disease mimics spontaneously occurring tumors in human patients), have demonstrated optimum therapeutic efficacy using tolerable doses. ${ }^{33-51}$ Our investigations, therefore, provide compelling rationale to develop phytochemical-embedded immunomodulatory nanomedicine agents for use in a wide array of applications in oncology. ${ }^{33-51}$

In view of the extraordinary importance of immunomodulatory intervention in treating mCRPC, we focused our attention on the creation of Mangiferin encapsulated gold nanoparticles (MGF-AuNPs). Mangiferin, used extensively in ancient medicine, is a glucose-functionalized xanthonoid found in large abundance in mangoes fruit peel. ${ }^{52}$ Several studies have shown that Mangiferin exerts antioxidant activities, inhibitory effects on type II $5 \alpha$-reductase in vitro, gastroprotective and also antidiabetic effects in rodents. ${ }^{53-57}$ Administration of Mangiferin in swiss mice have shown in vivo growth-inhibitory activity against ascitic fibrosarcoma. ${ }^{58}$ This phytochemical has demonstrated enhanced tumor cell cytotoxicity of the splenic cells and peritoneal macrophages of normal and tumor-bearing mice. ${ }^{59}$ Mechanistic investigations have revealed that Mangiferin induced decreased matrix metalloproteinase (MMP)-7 and -9 activities with concomitant reversal of epithelial-mesenchymal transition (EMT). ${ }^{60}$ There is conclusive evidence that the mechanism of modulation of MMP-7 and -9, and EMT is due to the innate ability of Mangiferin to inhibit $\beta$-catenin pathway. ${ }^{61,62}$ Enzymatic degradation, in vivo, has impeded the clinical applications of this important phytochemical in oncology.

Our hypothesis was that encapsulation of Mangiferin (MGF) on gold nanoparticles would create a new nanomedicine agent, MGF-AuNP, enabling improved cellular uptake of MGFAuNPs for exerting effective immunomodulatory intervention via targeting the tumor microenvironment. In the present article, we give a conceptual overview, on how a new generation of immunotherapeutic agent derived through green nanotechnology, integrating Mangiferin phytochemical onto well-defined gold nanoparticles (MGF-AuNPs), can be developed for use in prostate cancer therapy. Interestingly, combination of gold metal with phytochemicals has been used for over 5000 years in the Indian holistic Ayurvedic Medicine. Our green nanotechnology approach of encapsulating Mangiferin onto gold nanoparticles represents an integrative momentum to merge the best of two worlds of modern nanomedicine with the traditional Ayurvedic medicine.

We describe, herein, experimental evidence that Mangiferin functionalized gold nanoparticulate nanomedicine agent, (MGF-AuNPs), successfully manipulates the M1 and M2 polarization axis through two main approaches for applications in prostate cancer therapy: (i) specific interference with M2-like tumor associated macrophages (TAM) survival or inhibiting their signaling cascades and (ii) repolarization of tumor-promoting M2-like TAMs to a tumoricidal M1-like phenotype. We also describe evidence of immunomodulatory intervention of MGF-AuNPs in prostate cancers through observations of enhanced levels of anti-tumor 
cytokines, such as IL-12 and TNF- $\alpha$, with concomitant reductions in the levels of pro-tumor cytokines, such as IL-10 and IL-6. Additionally, we provide concrete details on cellular interrogation to establish that MGF-AuNPs target laminin receptors, over expressed on prostate tumor cells, thus presenting a compelling case for applications of this nanomedicine agent in the treatment of laminin receptor-positive human tumors for both diagnosis and therapy. Ability of MGF-AuNPs to target splenic macrophages is invoked via targeting of NF- $\kappa \mathrm{B}$ signaling pathway; and thus, resulting in reeducation/polarization of macrophages from pro-tumor M2 to anti-tumor M1 macrophages. Finally, therapeutic efficacy of MGF-AuNPs, in treating prostate cancer in vivo in tumor bearing mice, is described taking into consideration various modulatory interventions triggered by this green nanotechnology-based nanomedicine agent. Full mechanistic details of immunotherapeutic effects of MGF-AuNPs and how tumor microenvironment targeting ability of this nanomedicine agent will play a crucial role in prostate tumor therapy are described.

\section{Results and Discussion}

Green nanotechnological architecture and characterization of MGF-AuNPs. Mangiferin (1,3,6,7-tetrahydroxyxanthone-C2-D glucoside) is a polyphenol comprising of D-glucoside functionalized with a xanthone (Scheme 1). ${ }^{63}$ This phytochemical is found in abundance in the Anacardiaceae and Gentianaceae family of plant species especially in mango skin and honeybush tea. ${ }^{64}$ Following our pioneering efforts of using Phytochemicals of plants to produce tumor specific gold nanoparticles ${ }^{33-51}$, we have utilized a highly innovative green nanotechnology process to functionalize Mangiferin onto gold nanoparticles to produce Mangiferin encapsulated gold nanoparticles: MGF-AuNPs (Scheme 1). Antioxidant phytochemicals can act as electron reservoirs to transform metals into their corresponding nanoparticles. The high antioxidant capacity of Mangiferin, as reflected through its oxidation potential $(\mathrm{Epa}=0.32 \mathrm{~V})$, offered a unique opportunity to use this phytochemical to transform gold salt into the corresponding nanoparticles (AuNPs). We have now optimized a highly reproducible and a scalable process wherein interaction of appropriate amounts of Mangiferin with gold salt produced the corresponding phytochemical-encapsulated gold nanoparticles (MGF-AuNPs) in aqueous media (Scheme 1). The excess Mangiferin from the reaction mixture creates a robust encapsulation around gold nanoparticles thus eliminating the need for external chemical agents for stabilization against agglomeration of MGF-AuNPs (Figure 1). The green nanotechnology process, as depicted in Scheme 1 and Figure 2, offers a great example of a 'zero carbon footprint' process because, other than the gold salt, no other human-derived toxic chemicals were used in the overall production of MGF-AuNPs.

The MGF-AuNPs were characterized by a combination of techniques including UVVisible Spectrophotometry, Dynamic Light Scattering (DLS), Transmission Electron Microscopy (TEM) and Inductively Coupled Plasma Mass Spectrometry (ICP-MS). The UVvisible spectrophotometric analysis indicated a surface plasmon resonance (SPR) absorption at $\sim 535 \mathrm{~nm}$ (Figure 2A), thus inferring the successful synthesis of AuNPs. The core size of MGFAuNPs, obtained by TEM, indicated that the nanoparticles are spherical and nearly monodisperse with metallic core size of $35 \pm 2 \mathrm{~nm}$ (Figure 2). The results obtained by DLS measurements revealed that MGF-AuNPs exhibited a hydrodynamic size of $55 \pm 5 \mathrm{~nm}$ and a zeta potential $-40 \pm 2 \mathrm{mV}$ (Table 1). Hydrodynamic size of $55 \pm 5 \mathrm{~nm}$, which is greater than the core metallic size $(35 \pm 2 \mathrm{~nm})$ confirmed efficient encapsulation of Mangiferin around the gold nanoparticles. Preventing nanoparticles from aggregation is vitally important in the design and 
development of gold nanoparticles for biomedical applications. Kinetics of aggregation are dictated by electronic charge or through sterically demanding encapsulants. A significantly higher negative zeta potential $(\zeta)$ value of $-40 \pm 2 \mathrm{mV}$, as observed for MGF-AuNPs, clearly suggests that these nanoparticles possess optimum electrostatic repulsion to remain stable in solution for extended periods. Inductively coupled plasma mass spectrometry (ICP-MS) indicated that MGF-AuNPs contained 327 ppm of gold. Full Physicochemical parameters of MGF-AuNPs are summarized in Table 1.

Table 1: Physicochemical data parameters of MGF-AuNPs

\begin{tabular}{|c|c|c|c|c|c|}
\hline Sample & $\begin{array}{c}\text { Absorbance } \\
\text { (nm) }\end{array}$ & $\begin{array}{c}\text { Core size by } \\
\text { TEM (nm) }\end{array}$ & $\begin{array}{c}\text { Hydrodynamic } \\
\text { size by DLS (nm) }\end{array}$ & $\begin{array}{c}\text { Zeta potential } \\
\text { (mV) }\end{array}$ & $\begin{array}{c}\text { [Au] in AuNPs } \\
\text { (by ICP-MS, } \\
\text { ppm) }\end{array}$ \\
\hline MGF-AuNPs & $535 \mathrm{~nm}$ & $35 \pm 2$ & $55 \pm 5$ & $-40 \pm 2$ & 327 \\
\hline
\end{tabular}

Note. TEM: Transmission electron microscopy, ICP-MS: inductively coupled plasma mass spectrometry, DLS: dynamic light scattering, and MGF: Mangiferin.

Energy-dispersive X-ray spectroscopy (EDS) analysis of MGF-AuNPs provided information regarding the presence of gold (Figure 3). Additional characterization of MGFAuNPs was performed by recording the Powder X ray diffraction (PXRD) pattern (Figure 4). As shown in Figure 4, PXRD shows four peaks across the region of $28.5^{\circ}$ to $60.5^{\circ}$. The lowest angle peak at $29.5^{\circ}$ is a detector artifact which can be easily spotted from the X-ray diffraction photographs. The two major peaks at $38.32^{\circ}$ and $44.46^{\circ}$ match the known PXRD peaks for Au

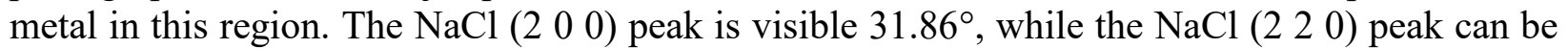
observed as a shoulder on the $\mathrm{Au}(200)$ peak with a maximum roughly at $45.60^{\circ}$.

In vitro stability study of MGF-AuNPs. The in vitro stability of MGF-AuNPs was evaluated by incubating solutions of nanoparticles with various biological media including $0.5 \%$ cysteine, $0.2 \mathrm{M}$ histidine, $0.5 \%$ human serum albumin (HSA), $0.5 \%$ bovine serum albumin (BSA), $1 \%$ $\mathrm{NaCl}$ and $\mathrm{pH} 7$ buffer solutions. Changes in SPR was used as a measure of stability in various media at different time points. ${ }^{35,39,41,43}$ As shown in Figure 1A, MGF-AuNPs exhibited no measurable changes in the position/shape of SPR, in various media throughout the measurement period. These observations suggest that the Mangiferin encapsulation creates a network of strongly hydrogen bonded corona around gold nanoparticles, thus affording excellent in vitro stability for over a week within various biological media (Figure 1A). In vitro stability of AuNPs, at dilutions that mimic in vivo biological conditions, is important for their applications in molecular imaging and therapy. ${ }^{33,34,37,41,42,45,50}$ Therefore, we have tested the stability of MGFAuNPs at various dilutions. As depicted in Figure 1B, MGF-AuNPs exhibited robust stability at extreme dilutions which are typical of cellular concentrations encountered under in vivo conditions.

Prostate tumor cell specificity and receptor mediated endocytosis of MGF-AuNPs. Previous studies from our laboratories have shown that polyphenolic structural motif of epigallocatechin 
gallate (EGCG) exhibit selective binding affinities (in the sub nanomolar ranges) with laminin receptors which are overexpressed in prostate and various other tumor cells. ${ }^{33,41} \mathrm{We}$ reasoned that the chemical structure of Mangiferin, which comprises of a glucose moiety (Scheme 1), should allow significantly efficient accumulation of MGF-AuNPs within tumor cells due to the Warburg effect. ${ }^{65,66} \mathrm{We}$ hypothesized that the Warburg effects, in conjunction with laminin receptor specificity of Mangiferin, are expected to provide selective and enhanced tumor accumulation of MGF-AuNPs. In order to establish laminin receptor specificity and high binding affinity of MGF-AuNPs toward laminin receptors, we have performed detailed mechanistic investigations on the endocytosis pathways of MGF-AuNPs in prostate tumor cells as discussed below.

It is well-known that prostate tumor cells overexpress laminin receptors (67 kDa LR) ${ }^{67}$ Laminin receptor is an important protein involved in cell adhesion to the basement membrane as well as in the signaling transduction following this binding event. ${ }^{68}$ In our experiments, we have probed the specificity of MGF-AuNPs toward laminin receptors that are over expressed in prostate tumor cells derived from human prostate tumors (PC-3 cells) (Figure 5 and Scheme 2). Briefly, PC-3 cells were treated with MGF-AuNPs, in two separate experiments involving the presence and absence of laminin receptor antibody (MLuC5), in our efforts to discern the laminin receptor mediated endocytosis of MGF-AuNPs. When the laminin receptors were not blocked by $\mathrm{MLuC}_{5}$ antibody, we observed very efficient endocytosis of MGF-AuNPs into PC-3 cells as shown in Figures 5B and 5E. We then saturated the laminin receptors on PC-3 cells using $\mathrm{MLuC}_{5}$ antibody and subsequently allowed these cells to interact with MGF-AuNPs. Microscopic analysis of these cells clearly revealed inhibition of MGF-AuNPs to internalize into PC-3 cells as shown in Figures 5C and 5F. We rationalize our findings on the basis that preincubation of PC-3 cells with $\mathrm{MLuC}_{5}$ antibody saturates Lam 67 receptors on prostate cancer cells and thus reduces or eliminates the ability of MGF-AuNPs to bind to laminin receptors on these cells. The above results, of pre- and post-blocking, of laminin receptors by $\mathrm{MLuC}_{5}$ antibody - taken together-unequivocally reveal that Mangiferin corona, on MGF-AuNPs, serves as a powerful laminin receptor targeting agent. These results, therefore, provide compelling evidence that MGF-AuNPs have the potential for use as tumor specific gold nanoparticles in molecular imaging and therapy of various laminin receptor-positive tumors.

In order to further establish tumor cell specificity of MGF-AuNPs, we have performed laminin receptor blocking experiments using a non-specific antibody, anti-fibronectin $\mathrm{AB}$ (Fn-3), and mouse IgG Isotype as a control. Our selection of these antibodies in blocking experiments is based on prior evidence that they do not have known specificity towards laminin receptors on PC-3 cells. In these experiments, PC-3 cells were pre-treated with both the antibodies separately followed by treatment with MGF-AuNPs for 60 min. Post incubation, the dark field microscopic and TEM analysis of tumor cells indicated that these antibodies failed to block the endocytosis of MGF-AuNPs within PC-3 cells (Figures 6E, 6F, 6I, 6J). In fact, the high propensity of endocytosis of MGF-AuNPs into PC-3 cells, post incubation with anti-fibronectin AB (Fn-3), and mouse IgG Isotype, was very similar to the results we have observed with the unblocked PC3 cells (Figure 6A). These detailed cellular interrogation investigations, as described above, clearly establish that MGF-AuNPs target laminin receptors on prostate tumor cells and thus reinforce their prospects for applications in the treatment of laminin receptor-positive human tumors for both diagnosis and therapy. 
Clathrin vs caveolae-mediated endocytosis and cell trafficking pathways of MGF-AuNPs. In order to understand the precise nature of the interaction MGF-AuNPs with prostate cellular (PC-3) membrane, we have explored further on the mode of endocytosis using two independent techniques involving dark field microscopy and transmission electron microscopy (TEM). The internalization and uptake of MGF-AuNPs with PC-3 cells were studied by incubating nanoparticles at various dilutions at select time points. Microscopic analysis of tumor cells, post incubation periods, revealed that MGF-AuNPs bind to prostate cell membrane within 30 min and internalize into the cells within 60 min of incubation time (Figure 7). Once the nanoparticles are accumulated on the cell membrane, these tumor cells appear to form a cavity like structure on the cell membrane to engulf the AuNPs (Figure 7C). These detailed time dependent studies suggested that MGF-AuNPs internalize into the tumor cells presumably through clathrin/caveolae mediated endocytosis. Generally, clathrin and caveolae mediated endocytosis follow receptor-mediated tumor-specific pathway whereas phagocytosis or pinocytosis follow non-specific pathways. In order to confirm that MGF-AuNPs are internalized through clathrin and/or caveolae mediated tumor specific endocytosis and not through the non-specific phagocytosis or pinocytosis pathways, we performed additional experiments involving preblocking clathrin coated pit using 'chlorprompazine' reagent followed by incubation with MGFAuNPs. Microscopic examinations of tumor cells from these clathrin blocking experiments revealed that significantly lower quantitates of MGF-AuNPs are internalized into tumor cells as shown in Figures 6B, 6C, 6G. These results clearly suggested that the mechanism of cell surface receptor uptake, and subsequent internalization of MGF-AuNPs, is mediated through clathrins. This is an important observation because clathrin mediated endocytosis are primarily responsible for subsequent intracellular downstream signaling and modulation of endocytic trafficking (discussed in subsequent sections). ${ }^{69,70}$ Our observations of clathrin-mediated endocytosis of MGF-AuNPs is of vital significance in prostate tumor therapy because recent investigations have shown that clathrin-mediated internalization of Cadherin-11 (Cad11) regulates surface trafficking of Cad11. It is well-known that Cadherin-11 cell adhesion molecule plays an important role in prostate cancer cell migration and that migratory function of Cad11 in prostate cancer cells is regulated through dynamic turnover of Cad11. ${ }^{70}$

We further focused our attention to test if MGF-AuNPs are also following caveolaemediated pathway for internalization within prostate tumor cells. Several pre-clinical and clinical investigations have suggested that expression of Caveolin-1 (Cav-1), an integral membrane protein expressed in two isoforms (Cav-1 $\alpha$ and $\mathrm{Cav}-1 \beta)$, as a significant prognostic marker for prostate cancer. ${ }^{71} \mathrm{Cav}-1$ is overexpressed in prostate cancer cells and is associated with the progression, cell survival and angiogenic activities of the disease. ${ }^{72}$ Therefore, we reasoned to explore if the efficient endocytosis of MGF-AuNPs, as depicted in Figures 6D, and 6H, is mediated through caveolae pathway. Towards this objective, we performed experiments to first block caveolae on prostate tumor cells by incubating them with Anti-Caveolin-1 antibody. Post blocking of caveolae on prostate tumor cells, we incubated these cells with MGF-AuNPs and subsequently performed detailed microscopic analysis. Dark field microscopic images of PC-3 cells with and without caveolae blocking, as shown in Figures 6D, and 6H, suggested little/no difference, between the pre and post caveolae blocking, in the amounts of nanoparticles that were internalized. These findings, therefore, revealed that the mechanism of endocytosis of MGFAuNPs in PC-3 cells is not mediated through caveolae expression and indeed occurs primarily through clathrin mediation as described above. 
Tumor targeting capabilities of MGF-AuNPs, as shown through extensive prostate tumor cell trafficking assays outlined above, prompted us to test the potential toxicity of these nanoparticles toward normal cells. The results are summarized in the following sections.

Interaction of MGF-AuNPs with normal cells. In order to elucidate that MGF-AuNPs are tumor cell specific and that they have minimal or no affinity toward normal cells, we have further evaluated cellular interaction of MGF-AuNPs using human aortic endothelial cells (HAECs). We hypothesized that MGF-AuNPs selectively target prostate tumor cells due to their overexpression of laminin receptors and that they cause minimal/no toxicity to normal cells because normal cells exhibit minimum laminin receptor density. ${ }^{73}$ Therefore, we incubated MGF-AuNPs with endothelial cells (HAECs) and looked for the uptake of gold nanoparticles in these cells through electron microscopy. The results presented in the Figure 8, confirmed that HAECs showed minimum uptake of MGF-AuNPs at the same dose and time point $(41 \mu \mathrm{M} ; 60$ min incubation) as was used for similar experiments with prostate tumor cells (PC-3). These results are of profound importance in the context of various applications of MGF-AuNPs as tumor specific therapeutic agent with minimal/no toxicity to normal cells.

These detailed cellular interrogation investigations, as described above, have clearly established that MGF-AuNPs target laminin receptors on prostate tumor cells and that they exhibit minimal/no toxicity to normal cells. Exploring the effects of MGF-AuNPs on prostate tumor and normal HAECs cell viability was the next logical step in our quest to validate the applicability of MGF-AuNPs as a nanomedicine agent for treating prostate cancer.

Effects of MGF-AuNPs on prostate tumor and normal HAECs cell viability. We have performed MTT assays to evaluate viability of prostate tumor and HAECs cells upon treatment with MGF-AuNPs. Choice of PC-3 cells was rationalized based on their innate metastatic nature. Serial dilutions of MGF-AuNPs were prepared in RPMI media to treat with PC-3 cells. The cell viability profiles, as shown in Figure 9A, inferred that these nanoparticles exhibited dose dependent efficacy for causing the death of cancer cells. Figure 9A depicts increased reduction in cancer cell viability with increasing concentrations of the MGF-AuNPs agent over a period of 48 and 72 hours. At each of the concentrations, we observed reduced cell viability as compared to the control untreated, and a significant reduction of tumor cells was noted at a concentration of $165 \mu \mathrm{M}$ and beyond.

In summary, MTT cell viability assay, for normal cells (Figure 9B) and prostate tumor cells (Figure 9A), taken together, demonstrated that MGF-AuNPs presented limited/no toxicity toward normal cells whereas dose limiting selective toxicity was seen on tumor cells (Figures 9).

Evaluation of induction of apoptotic vs necrotic cancer cell death patterns of MGF-AuNPs on PC-3 cells. We wanted to test if the mechanism of tumor cell death, when MGF-AuNPs interacted with PC-3 cells, is driven through a regulated programmed cell death (apoptosis), or through a passive, uncontrolled necrosis course. Apoptotic cells are measured by their cell membrane disruption, chromatic condensation, and DNA degradation which leads to cell death. In our assays, we used flow cytometry and fluorescent microscope techniques to visualize patterns of early and late apoptosis by PI and FITC-Annx V staining. Cells with early apoptosis are FITC (+ve) and PI (-ve), whereas cells at late apoptotic stages are FITC (+ve) and PI (+ve). Our results have confirmed that PC-3 cells treated with MGF-AuNPs showed $40 \%$ total cell death including early and late stage apoptosis at a dose of $83 \mu \mathrm{M}$ (Figure 10A). In the untreated 
control group, cell death was found to be $14 \%$, which corresponds to the normal programmed cell death. These results provide important insights that MGF-AuNPs exert apoptotic influence on PC-3 cells in tumor selective therapy. We have further verified, the Annexin V/PI assay results, through careful observations of cellular morphology of PC-3 cells upon treatment with MGF-AuNPs. The results presented in Figure 10B indicated that cells treated with MGF-AuNPs exhibited significantly more apoptosis compared to untreated control cells. Early apoptotic cells are colored in green whereas late stage apoptotic and/or necrotic cells are colored in red (Figure 10B). It may be discerned, from Figures 10A and 10B that more cells were found in early and late apoptotic stages, in the MGF-AuNPs treatment group, as compared to the untreated control group. It is also significant to recognize that MGF-AuNPs, at the dose of $83 \mu \mathrm{M}$, showed almost similar pattern of apoptotic cells death as was observed upon treatment of cancer cells with the standard drug 'Staurosporine' (Figure 10B). In summary, all our results, taken together, unequivocally, confirmed that MGF conjugated-AuNPs induced apoptosis of cancer cells through early-stage apoptotic phase, and finally resulting in effective programmed cancer cell death.

Anti-angiogenesis activity of MGF-AuNPs. Angiogenesis plays a vital role in the overall growth process of cancer as it dictates the migration and differentiation of endothelial cells, which line the inside wall of blood vessels. ${ }^{74}$ Chemical signals in the body control the rapidity of angiogenesis by providing efficient blood supply that stimulate angiogenesis as well as stimulate nearby normal cells to produce angiogenesis signaling molecules. ${ }^{75}$ Therefore, angiogenesis inhibiting agents are pivotal in the effective treatment of various cancers, particularly the solid tumors. We have, therefore, performed additional studies to elucidate the anti-angiogenesis capabilities of MGF-AuNPs through capillary tube structures formation assay. Phase contrast microscopic images, as depicted in Figure 10C, clearly showed that the HAECs cells, preincubated with MGF-AuNPs nanomedicine agent, at 41 and $83 \mu \mathrm{M}$ doses effectively inhibited the formation of capillaries as compared to the control untreated group, where the complete vasculature structure was seen intact (Figure 10C). Angiogenesis plays a critical role in the growth of cancer because solid tumors need a blood supply if they are to grow beyond a few millimeters in size. ${ }^{76}$ Tumors can actually cause this blood supply to form by giving off chemical signals that stimulate angiogenesis. Tumors can also stimulate nearby normal cells to produce angiogenesis signaling molecules. ${ }^{75}$ Vinblastine has emerged as an effective microtubule destabilizing agent because of its ability to target tubulin, thus inhibiting its polymerization and the subsequent association of microtubules. The superior antiangiogenic features of vinblastine restrain the tumor growth while decelerating malignant angiogenesis in vast majority of human cancers. ${ }^{77,78}$ Therefore, we have compared the antiangiogenic characteristics of MGF-AuNPs with the FDA approved vinblastine. Our results, as depicted in Figure 10C, compellingly infer that the anti-angiogenesis effects of MGF-AuNPs are comparable with the FDA approved drug vinblastine. These findings provide compelling evidence on the vast potential of MGF-AuNPs for use as an anti-angiogenesis agent in oncology.

Role of nuclear factor kappa $B$ (NF-кB) transcription factor in prostate cancer. In the context of prostate cancer, several clinical investigations, involving human prostate cancer patients, have shown strong correlations between increased frequency of NF- $\kappa \mathrm{B}$ p65 and a risk of disease progression. ${ }^{79}$ Indeed, the identification of patients with high-risk prostate cancer (PC) and its direct association with the nuclear localization of NF- $\kappa \mathrm{B}$ p65, from cohorts of patients, 
has generated considerable interest in the tremendous prognostic clinical value of this cell signaling pathway as a potential prognostic parameter in gauging treatment outcomes of advanced stage prostate cancer patients. ${ }^{80}$ Therefore, we have probed the potential utility of MGF-AuNPs as a NF- $\kappa$ B targeting agent through interactions with PC-3 prostate tumor cells from the human prostate tumor origin.

MGF-AuNPs target nuclear factor kappa B (NF-кB) transcription factor. Nuclear factor kappa B (NF- $\kappa \mathrm{B})$ constitute a family of genes acting in concert in malignant tumor invasion, migration and metastasis, of various human cancers including breast, colon, lung, oral, pancreatic, and prostate cancers. ${ }^{81,82}$ Several investigations have inferred that (NF- $\left.\kappa \mathrm{B}\right)$ activation is directly responsible for the cross talk between inflammation and cancer progression. ${ }^{83}$ The remarkable interrelationship of NF- $\mathrm{NB}$ activation to tumor progression - through a combination of processes including tumor cell proliferation, retarding apoptosis, accelerating angiogenesis, promoting pro-tumor macrophages - singularly and collectively underscore the importance of developing new therapeutic agents that target NF- $\mathrm{BB}$ both for the prevention as well as for the treatment of various human cancers. ${ }^{83,84}$ We have, therefore, undertaken evaluations to examine if MGF-AuNPs can efficiently suppress the activation of NF- $\kappa \mathrm{B}$ in tumor cells. Our investigations entailed seeding PC-3 cells into 6 well plates with subsequent treatment with MGF-AuNPs. These MGF-AuNPs-treated cells were subsequently post-treated with tumor necrosis factor alpha (TNF- $\alpha$ ) for another $30 \mathrm{~min}$ at $37^{\circ} \mathrm{C}$. TNF- $\alpha$, is a multifunctional proinflammatory cytokine that belongs to the tumor necrosis factor superfamily. ${ }^{85}$ The control group PC-3 cells were incubated with TNF- $\alpha$ only to stimulate NF- $\kappa B .{ }^{86,87}$ Quantification of NF- $\kappa B$ suppressive effects of MGF-AuNPs was carried out using flow cytometry in comparison with the controls. The flow cytometry analysis, as shown in Figure 11, indicated that MGF-AuNPs effectively blocked the TNF- $\alpha$-induced-NF-kB activation in the PC-3 cells, which were pretreated with nanomedicine agent, with subsequent post treatment with TNF- $\alpha$. Images depicted in Figure 11 further confirmed that the NF- $\kappa B$ levels were indeed significantly higher in the PC-3 control group — which were not treated with MGF-AuNPs (Figure 11). These studies highlight two important experimental findings that MGF-AuNPs can be used: (i) for the inhibition of NF- $\mathrm{NB}$ signaling thereby transferring signals to the nucleus to induce corresponding gene expression, to control excessive cell proliferation, reduce/eliminate apoptotic resistanceall resulting in anti-angiogenesis, inhibiting invasion, and thus to effectively control/eliminate metastasis; and (ii) in the overall design of new targeted therapeutics aimed at cancer prevention and therapy.

TNF- $\alpha$ has gained a ubiquitous "yin and yang" role in cancer development and metastases. It is well-known that TNF- $\alpha$ released from macrophages activates NF- $\kappa \mathrm{B}$-mediated signaling pathway in various cancers - thus playing a major role in cancer progression and metastasis. ${ }^{85-88}$ Tumor microenvironment is highly dynamic in cell-to-cell crosstalk between $\mathrm{NF}-\kappa \mathrm{B}$ and other signaling pathways. Such crosstalk feedback loops modulate the inflammatory response in macrophages by altering NF- $\mathrm{BB}$ activation. ${ }^{89}$ Encouraged by the NF- $\mathrm{BB}$ targeting ability of MGF-AuNPs, we reasoned the logistics of the role of this nanomedicine agent in targeting tumor microenvironment. A strong rationale for such investigations stemmed from the fact that tumor-associated macrophages (TAMs) engineer regulation of cancer growth and metastases through alterations of tumor cell proliferation, migration, invasion, angiogenesis, and immunosuppression. Immune cells outside the tumors produce pro-inflammatory cytokines to activate tumor NF- $\mathrm{kB}$ pathway of tumor cells and also tumor-infiltrating cells such as 
macrophages and myeloid-derived suppressor cells (MDSCs) - all resulting in a tumorpermissive environment for the growth and metastasis.

In the following sections, we will describe the key findings of the innate ability of MGFAuNPs to target macrophages and subsequently on how the macrophage affinity of this nanomedicine agent would translate into the design of a new immunomodulatory prostate cancer therapeutic agent.

Target specificity of MGF-AuNPs toward macrophages. Macrophages are classified as nonneoplastic cells with pro-tumor or anti-tumor phenotypes depending on their anatomical location, and the physiological context. Classically activated macrophages (referred to as M1) and alternatively activated macrophages (referred to as M2) fit two extremes within the spectrum of the macrophage phenotypes. ${ }^{90}$ Tumor-associated macrophages (TAMs) closely resemble "alternative" (M2) macrophages. ${ }^{91}$ M1 macrophages are recognized as classically activated macrophages that can phagocytosis pathogens and exert tumoricidal activity through activation of antitumor activity primarily by IL-12-dependent natural killer (NK) cell recruitment. On the other hand, proliferating tumors in humans exhibit polarized M2 phenotype that are directly involved in tumor metastasis, and ultimately contributing to drug resistance of the disease. ${ }^{92}$ Tumor associated macrophages often express M2-like phenotype with high IL-10, high arginase1 and low IL-12 - all contributing to pro-tumorigenic activities. In the context of prostate cancer, there is considerable evidence supporting macrophage infiltrations (inflammation) which are associated with especially advanced stages of prostate cancer. In fact, castrated tumors possess more pro-tumorigenic M2 macrophage phenotype thus inducing the onset of immunosuppressive state..$^{93-96}$

It is also important to note that drugs capable of targeting NF- $\kappa \mathrm{B}$ signaling in TAMs can reprogram macrophages from the pro-tumor M2 to an anti-tumor M1 phenotype. This process within the TAMs promotes regression of advanced tumors by induction of macrophage tumoricidal activity and activation of antitumor activity through IL-12-dependent NK cell recruitment. ${ }^{97,98}$ Given the importance of M2 to M1 macrophage reeducation and the established role of MGF-AuNPs in targeting NF- $\mathrm{B}$ signaling (Figure 12), the logical next step was to evaluate the macrophage targeting ability of this nanomedicine agent, especially to explore its capability in transforming pro-tumor M2 to an anti-tumor M1 phenotype within TAMs.

In our initial experiments, we treated the RAW 264.7 macrophages with MGF-AuNPs and evaluated for the endocytosis of these nanoparticles in macrophage cells. As shown in Figure 13, indeed, MGF-AuNPs displayed excellent affinity and propensity to internalize within macrophages through phagocytosis. It is important to note that comparison of images (Figure $13 \mathrm{~A}$ and 13B), demonstrates that, under similar experimental conditions of using MGF-AuNPs $(40 \mu \mathrm{M})$ incubated for $60 \mathrm{~min}$, macrophages assimilated higher payloads of MGF-AuNPs as compared to PC-3 cells. Macrophages phagocytized MGF-AuNPs efficiently, whereas the PC-3 cells, which use laminin receptor-mediation as the primary process to internalize these nanoparticles, exhibited significantly lower propensity for internalization of this nanomedicine agent. Having established the macrophage-avidity of MGF-AuNPs, we turned our attention in testing the ability of these nanoparticles in inhibiting NF- $\kappa \mathrm{B}$ phosphorylation in macrophages.

MGF-AuNPs inhibit NF- $\kappa$ B phosphorylation in macrophages. NF- $\kappa B$ is a transcription factor that resides in IкB kinase (IKK) complex located in the cytoplasm along with inhibitor of NF- $\kappa \mathrm{B}$ proteins $(\mathrm{I} \kappa \mathrm{Bs}), \mathrm{NF}-\kappa \mathrm{B}$ activation is stimulated by $\mathrm{TNF}-\alpha$, or other cell stressors which then 
leads to NF- $\mathrm{B}$ phosphorylation and translocation to the nucleus. ${ }^{80}$ This directly influences the transcription of pro-tumor genes in cancer cells and macrophages. The rationale for our investigations is that the NF- $\mathrm{KB}$ intervention in macrophages can polarize macrophages to the anti-tumor M1 phenotype to eliminate tumors. ${ }^{91}$ In order to evaluate the ability of MGF-AuNPs to induce NF- $\mathrm{B}$ inhibition in macrophages, we pretreated RAW 264.7 with either MGF-AuNPs or starch encapsulated AuNPs (Starch-AuNPs: S-AuNPs served as a control AuNPs). Our experimental findings revealed that MGF-AuNPs inhibited RANKL induced NF- $\kappa \mathrm{B}$ in macrophages (Figure 12E). Our results indicate that MGF-AuNPs induce polarization of macrophages to anti-tumor phenotype by inhibiting NF- $\kappa \mathrm{B}$ phosphorylation. ${ }^{91} \mathrm{NF}-\kappa \mathrm{B}$ is frequently dysregulated during the onset and progression of cancers which makes this protein critical in the manifestation of these malignancies. Intervention of the overactive NF- $\kappa \mathrm{B}$ in TAMs using nanomedicine agent which passively targets these macrophages might prove to be decisive step in controlling prostate gland related malignancies. ${ }^{93}$ Concurrently, our investigations also focused on the capabilities of MGF-AuNPs, to induce polarization of macrophages to the anti-tumor or M1 phenotype.

MGF-AuNPs, but not S-AuNPs, polarize macrophages to anti-tumor or M1 phenotype. Tumor microenvironment modifies the macrophages which then aid in the progression of these tumors. ${ }^{99}$ The modified macrophages or TAMs have reduced antigen presentation ability and produce elevated levels of immunosuppressive cytokines such as IL-10. ${ }^{99}$ The macrophages within the tumor microenvironment also produce increased levels of antiangiogenic cytokines such as IL-6. ${ }^{100}$ In order to understand the effects of MGF-AuNPs on macrophages, we have investigated the expression of macrophage polarizing cytokines such as IL-12, IL-10, IL-6, and TNF- $\alpha$ upon treating RAW 264.7 macrophages with MGF-AuNPs. In RAW 264.7 macrophages, which were treated with MGF-AuNPs, our experimental findings conclusively demonstrated elevated levels of anti-tumor cytokines such as IL-12 and TNF- $\alpha$, while reducing the levels of pro-tumor cytokines such as IL-10 and IL-6 (Figure 12A, D). In contrast, the results from similar investigations using the starch-stabilized gold nanoparticles (S-AuNPs), we observed no macrophage targeting capability and no influence in elevating the levels of anti-tumor cytokines such as IL-12 and TNF- $\alpha$ (Figure 12). These results suggested the potential immunotherapeutic role of MGF-AuNPs and, therefore, prompted us to further probe into capabilities of this nanomedicine agent for modifying macrophages.

In order to evaluate if MGF-AuNPs can reprogram M2 macrophages into the therapeutically desirable anti-cancer M1 phenotype, we cocultured MGF-AuNPs-pretreated macrophages with prostate tumor cells (PC-3) and then looked for tumor proliferation differences between this group and the control PC-3 cells which were directly treated with naïve macrophages only. We found that macrophages transfected with the MGF-AuNPs agent displayed gene expression profiles similar to anti-tumor M1 phenotype. This observation is consistent with the significant inhibition in the proliferation of tumor cells. However, PC-3 cells that were cocultured with naïve macrophages failed to reduce the proliferation of PC-3 cells (Figure 14). These data suggest that MGF-AuNPs-mediate anti-tumor phenotype to macrophages expression and therefore warranted further investigations on whether this nanomedicine agent would promote elevation in the levels of anti-tumor cytokines such as IL-12 and TNF- $\alpha$ while reducing the levels of pro-tumor cytokines such as IL-10 and IL-6. With this objective in mind, we incubated MGF-AuNPs with RAW 264.7 macrophages and analyzed the levels of various pro-tumor and anti-tumor cytokines. Real-time PCR (Quantitative-PCR) showed robust increase 
in anti-tumor (pro-inflammatory) cytokines IL-12 (10-fold higher) and TNF-a (50-fold higher), while reducing the levels of pro-tumor cytokines such as IL-10 and IL-6 in macrophages treated with MGF-AuNPs. Similar experiments using the starch-stabilized gold nanoparticles (S-AuNPs) control showed no effects toward enhancing anti-tumor cytokines in the treated macrophage cells (Figure 12). Tumor microenvironment modifies the macrophages which then aid in the progression of these tumors. ${ }^{94}$ The modified macrophages or TAMs have reduced antigen presentation ability and produce elevated levels of immunosuppressive cytokines such as IL10. ${ }^{99}$ The macrophages within the tumor microenvironment also produce increased levels of antiangiogenic cytokines such as IL-6. Therefore, our results which demonstrate the ability of MGF-AuNPs in promoting the levels of anti-tumor cytokines, such as IL-12 and TNF- $\alpha$, while reducing the levels of pro-tumor cytokines, such as IL-10 and IL-6, are significant toward proving the immunomodulatory intervention in prostate cancer therapy (Scheme 3).

TAMs which originate from resident macrophages from the bone marrow and spleen are key tumor stromal cell types and play a critical role in tumor survival, growth, and metastasis. ${ }^{101,102}$ Several investigations have confirmed spleen macrophages $(\mathrm{M} \varphi)$ as the key TAM precursors, where macrophages maintain hematopoietic steady state by engulfment of neutrophils and eosinophils. ${ }^{103}$ Tumor microenvironment modifies the macrophages which then aid in the progression of these tumors. The modified macrophages or TAMs have reduced antigen presentation ability and produce elevated levels of immunosuppressive cytokines such as IL-10. The macrophages within the tumor microenvironment also produce increased level of antiangiogenic cytokines such as IL-6. It is important to understand how enhanced retention of MGF-AuNPs in spleen affects macrophage function. In order to explore the ability of MGFAuNPs to target splenic macrophages, we have undertaken a detailed biodistribution study of MGF-AuNPs in normal mice as discussed below.

Targeting splenic macrophages. Spleen is a critical secondary lymphoid organ showing abundance of B cells, T cells, NK cells, and is also a reservoir of mononuclear phagocyte system (MPS), mainly resident macrophages. ${ }^{104}$ Recent investigations have shown that the local and systemic immune response to cancer increases by the ability of drugs or nanoparticles to target splenic macrophages which comprise mostly of M2-like pro-tumor macrophages. ${ }^{105}$ Macrophage position and function in splenic domains confer them unique phenotypes. ${ }^{106}$ Suzuki et al have shown that Gemcitabine selectively eliminates splenic $\mathrm{Gr}-1^{+} / \mathrm{CD} 11 \mathrm{~b}^{+}$myeloid suppressor cells in tumor-bearing animals and enhances antitumor immune activity. ${ }^{107}$ Indeed, the superior antitumoral efficacy of Trabectedin (Yondelis ${ }^{\circledR}$ ) has been attributed to the ability of this drug to target splenic macrophages thus exerting TAM selective cytotoxic activity towards Ly6C ${ }^{\text {high }}$ monocytes in circulation and in the spleen. ${ }^{108}$ In order to evaluate further on the macrophage targeting ability and immunomodulatory characteristics of MGF-AuNPs, we have performed systemic administration via intravenous injection in mice-all aimed at testing if this nanomedicine agent is effective in targeting splenic macrophages. We discuss here compelling evidence from murine models of cancer in support of macrophage-targeted intervention strategies with the potential of MGF-AuNPs for use in dramatically reducing prostate and various other cancer morbidities through immunomodulatory mechanisms.

In order to understand the pharmacokinetics/ pharmacodynamics of MGF-AuNPs, we have evaluated the uptake of gold nanoparticles, in vivo, in normal mice as well as in prostate tumor-bearing mice. These studies were performed by first producing the radioactive equivalent of MGF-AuNPs using ${ }^{198} \mathrm{Au}$ isotope because the gamma $(0.411 \mathrm{MeV})$ emission of ${ }^{198} \mathrm{Au}$ isotope 
allows scintigraphic counting of radioactivity of gold for accurate estimation of the nanomedicine agent in various organs. ${ }^{39}$ Post administration of radioactive MGF- ${ }^{198} \mathrm{AuNPs}$, in normal mice, through intravenous delivery, we analyzed for the presence of gold in various organs at various time points using scintigraphy counting (Figure 15A). ${ }^{39}$ Our results, as shown in Figure 15A, showed a significant uptake of MGF- ${ }^{198} \mathrm{AuNP}$ in spleen. This observation is clearly in stark contrast to a vast variety of gold nanoparticles, which generally show hepatobiliary uptake in liver. ${ }^{34,109}$ Together with the results, as discussed above, on the ability of MGF-AuNPs to target NF- $\kappa \mathrm{B}$; and to reeducate/ polarize from pro-tumor M2 to anti-tumor M1 macrophages - the high uptake of MGF-198AuNPs in spleen (Figure 15) is consistent with the ability of this agent to target splenic macrophages.

It may be noted that the mode of delivery, going from the intravenous to intraperitoneal, did not reduce the uptake of MGF-AuNPs in splenic macrophages. The preferential accumulation in liver and spleen invoked the possibility of this nanomedicine agent to target splenic macrophages. In order to elucidate if the uptake of MGF-AuNPs in spleen is indeed of splenic macrophage origin, we have performed further in vivo investigations using severely combine immune-deficient (SCID) mice. The rationale for this animal model is based on the fact that SCID mice manifest active macrophages while lacking in $\mathrm{T}$ and $\mathrm{B}$ cells. ${ }^{110}$ Before moving onto in vivo tumor studies with MGF-AuNPs, we tested the toxicity of this nanomedicine agent on SCID mice. The doses of MGF-AuNPs, at which prostate tumors are suppressed in vivo, caused no systemic toxicity in normal mice as elucidated through detailed toxicity studies (see supplementary materials section). Intraperitoneal administration of MGF-AuNPs in SCID mice resulted in preferential accumulation in liver and spleen which further suggested that MGFAuNPs might be assimilated by splenic macrophages (Figure 15). Macrophages play important role in tumor development by supporting vascularization of tumors as well as inhibition of subsequent generation of tumor specific cytokines. It is well-known that macrophages migrate to spleens to phagocytize dead RBCs, and therefore, the accumulation of MGF-AuNPs in spleen, as observed, suggests the selective accumulation of this nanomedicine agent in the macrophages.

The in vivo distribution of MGF-AuNPs in SCID and normal mice, which revealed high percentage of accumulation of MGF-AuNPs in spleen, suggested possible macrophage-based internalization. Macrophage position and function in splenic domains confer them unique phenotypes thus corroborating tumor killing properties of MGF-AuNPs-pretreated macrophage cells, as observed in the co-culture experiments involving PC-3 cells (Figure 14). These experimental findings are of significance because they lend experimental evidence on the unique characteristic of this nanomedicine agent to exploit trophic macrophages to subvert innate and adaptive immune responses capable of destroying malignant cells.

Targeting NF- $\mathrm{BB}$ signaling pathway, induction of polarization of macrophages to antitumor phenotype by inhibiting NF- $\mathrm{B}$ phosphorylation, ability to promote the levels of antitumor cytokines, such as IL-12 and TNF- $\alpha$, as discussed above-individually and collectivelyinfer the immunomodulatory features of MGF-AuNPs. In vivo therapeutic efficacy studies of MGF-AuNPs in tumor model was an obvious next step to evaluate whether the various immunomodulatory parameters, as observed in vitro, would be translated under the more complex in vivo tumor profiles in tumor bearing mice. We have therefore, undertaken detailed therapeutic efficacy of MGF-AuNPs in prostate tumor bearing SCID mice as discussed below.

Therapeutic efficacy of MGF-AuNPs in treating prostate tumor. We have used severely compromised immunodeficient (SCID) male mice bearing a flank model of human prostate 
cancer, derived from a subcutaneous implant of 10 million PC-3 cells, for the therapeutic efficacy and pharmacokinetic studies. In our evaluations, unilateral solid tumors were allowed to grow for three weeks, and animals were randomized (denoted Day 0) into control and treatment groups $(\mathrm{n}=7)$ with no significant differences in tumor volume. In vivo dosing involved administering on day 0 three doses of MGF-AuNP $(0.5 \mathrm{mg} / \mathrm{kg}$ bw, $1.0 \mathrm{mg} / \mathrm{kg}$ bw and $1.5 \mathrm{mg} / \mathrm{kg}$ bw-in $100 \mu \mathrm{L}$ Dulbecco's PBS) intraperitoneally, while the control SCID mice received only $100 \mu \mathrm{L}$ Dulbecco's PBS/saline. This treatment regimen was performed twice per week. Tumors were then measured twice each week until the end of the study (Day 42). Figure 16 shows results from the MGF-AuNPs-treated human prostate cancer bearing SCID mice. Within two weeks (Day 14), tumor growth in the treated animals started slowing down with respect to the control animals. Day 17, post administration of MGF-AuNPs $(1.5 \mathrm{mg} / \mathrm{kg} \mathrm{bw})$, tumor volumes were twofold lower $(p<0.005)$ for treated animals as compared to the control group. Three weeks, post administration of after MGF-AuNPs $(1.5 \mathrm{mg} / \mathrm{kg} \mathrm{bw})$, tumor volumes for the control animals were fully six-fold greater with respect to those for the MGF-AuNPs-treated group $(p<0.0001$; $\left.0.37 \pm 0.05 v s .0 .06 \pm 0.02 \mathrm{~cm}^{3}\right)$ - suggesting $>85 \%$ reduction in the overall tumor volume for the treated group. This significant therapeutic effect was maintained throughout the 42 days long study. Tumors harvested from the treatment group consisted largely of necrotic tissue, indicating extensive death of tumor cells.

Interestingly, biodistribution studies suggested that very low percentage of MGF-AuNPs reached the tumor tissue while most of the therapeutic agent was found in the spleen. It is important to note that SCID mice lack T and B cells which are needed for the growth of human prostate tumors. In this context, the remarkable therapeutic efficacy, as shown in Figure 16, therefore, suggests the ability of MGF-AuNPs to target the tumor microenvironment and, thus ensue modification of macrophages as the primary mode of therapeutic action of this nanomedicine agent.

The compelling therapeutic efficacy data, as summarized above, was further corroborated through evaluation of angiogenesis inhibitory effects of MGF-AuNPs in vivo. Angiogenesis is ubiquitous in tumor growth, invasion, progression, and metastasis of a vast majority of human cancers. ${ }^{11}$ Therefore, targeting this process may potentially halt the growth and spread of cancers. ${ }^{112}$ Some of the prominent FDA approved angiogenesis inhibitors currently used in cancer therapy (with their mode of action) include: Bevacizumab (VEGF-A antibody); Ramucirumab (VEGFR2 antibody); Sunitinib (Tyrosine Kinase Inhibitor); Sorafenib (Tyrosine Kinase Inhibitor); Pazopanib (Tyrosine Kinase Inhibitor); Lenvatinib (Tyrosine Kinase Inhibitor); and Cabozantinib (Tyrosine Kinase Inhibitor). ${ }^{12}$ Angiogenesis inhibitors act through direct interference in blood vessel growth. A well-established mode of action of angiogenesis inhibitors manifests binding to VEGF and/or its receptors. ${ }^{113}$ Angiogenesis inhibitors are also known to bind to various surface receptors of endothelial cells or they block blood vessel growth through strong and selective binding interactions with proteins in the downstream signaling pathways. Our rationale behind these studies stemmed from a variety of immunomodulatory features of MGF-AuNPs, as discussed in the preceding sections. There is considerable experimental evidence supporting that angiogenesis inhibitors are immunomodulatory and are capable of suppressing tumor growth. ${ }^{114}$

Inhibition of angiogenesis in vivo. The compelling immunomodulatory effects, in controlling growth of tumors in vivo, prompted us to investigate the effects of MGF-AuNPs on angiogenesis in tumor tissues obtained through in vivo therapeutic efficacy experiments as discussed above. 
These studies were performed through immunostaining using the CD31 antibody because it has high specific affinity for vascular endothelial cells. Twelve fields (at 400x), from each prostate tumor xenografts, were analyzed to determine the average number of vessels per field (microvessel density $=$ MVD). The findings from studies indicated a significant reduction of MVD in samples collected from the MGF-AuNPs $(1.5 \mathrm{mg} / \mathrm{kg} \mathrm{bw})$ treated animals as compared to the control group (saline treated) (Figure S1C). Manifestation of angiogenesis in MGF-AuNPstreated tumor tissues, taken in concurrence with a plethora of immunomodulatory data discussed above, infers the immunomodulatory angiogenesis inhibitor characteristics of this nanomedicine agent exerting stimulatory effects on the immune system.

\section{Conclusions}

Our studies lend credible experimental evidence demonstrating that inhibition of the receptor activator of NF- $\mathrm{BB}$, by the new nanomedicine agent MGF-AuNPs, prevents prostate cancer development. The ability of MGF-AuNPs to target NF- $\kappa \mathrm{B}$ signaling pathway will provide an attractive therapeutic strategy for the treatment of various forms of advanced cancers. As a vast majority of patients with solid tumors require therapeutic approaches with capabilities to reprogram the local immunosuppressive tumor milieu in order to revive antitumor immunity, detailed immunotherapeutic investigations, and results reported herein, provide compelling evidence on macrophage targeting abilities of the new MGF-AuNP nanoceutical. Our preclinical in vitro and in vivo studies have shown that MGF-AuNPs effectively target tumorassociated macrophages (TAMs) which abundantly infiltrate most solid tumors. TAMs-targeting strategies of MGF-AuNPs have been effectively used to initiate macrophage re-education from pro tumor M2 macrophages to antitumor M1 phenotype-thus eliminating cancer cells, restrict tumor growth and metastasis. Overall, our green nanotechnology discoveries, which have resulted in the development of a new generation of phytochemical-encapsulated nanomedicine agent (MGF-AuNP), provide further new insights on the therapeutic potential of TAM targeting nanoceuticals to improve immunotherapies.

\section{Methods}

Materials. Mangiferin, Sodium tetrachloroaurate (III) dihydrate, MTT (3-(4, 5-dimethyl thiazol2-yl)-2, 5-diphenyl tetrazolium), dynasore reagent and Chlorpromazine (CPZ) were obtained from Sigma (St. Louis, MO, USA). RPMI, fetal calf serum, TryplE, Trypan blue, DAPI (4',6diamidino-2-phenylindole), 2,7-dichlorofluorescin diacetate (DCFH-DA), mouse IgG isotype control, and laminin receptor antibody $\left(\mathrm{MLuC}_{5}\right)$ were obtained from ThermoFisher Scientific, USA. FITC Annexin V Apoptosis Detection Kit was obtained from BD Pharmingen, USA. X-22 anti-clathrin antibody (ab2731), anti-Caveolin-1 antibody (ab2910), anti-fibronectin antibody (ab18265), and in vitro angiogenesis assay kits (ab204726) were obtained from Abcam, USA. GFP-CERTIFIED ${ }^{\circledR}$ Apoptosis/ Necrosis detection kit (ENZ-51002) was obtained from Enzo Life Sciences, Inc., USA. Phospho-NF-кB p65 (Ser536) (93H1) Rabbit mAb (Alexa Fluor®488 Conjugate) Kit was obtained from Cell Signaling Technology, USA. Double distilled water was used throughout the experiment.

Cell lines. The human prostate cancer (PC-3), human aortic endothelial cells (HAECs), and mouse macrophages (RAW 264.7) cell lines were obtained from the American Type Culture Collection (ATCC; Manassas, VA), and cultured by the University of Missouri Cell and Immunobiology Core facility using procedures recommended by ATCC. 
Characterization of nanoparticles by TEM. Transmission electron microscopic (TEM) images were obtained on a JEOL 1400 TEM (JEOL, LTE, Tokyo, Japan) available at the University of Missouri Electron Microscopy Core facility. The absorption measurements were attained by UVVis spectrophotometer (Varian Cary 50 conc, USA). The hydrodynamic size and zeta potential were obtained using a Zetasizer Nano S90 (Malvern Instruments Ltd. USA). The concentration of gold metal in nanoparticles were estimated by inductively coupled plasma mass spectrometry (ICP-MS), Perkin Elmer, MA, USA. The presence of gold metal in MGF-AuNPs was characterized using energy-dispersive X-ray spectroscopy (EDS) ${ }^{115\{\text { Brun, } 2010 \# 10}$

Characterization of nanoparticles by X-ray diffraction technique. Liquid nanoparticle dispersions in water were spread out on disposable glass slides and allowed to evaporate overnight under ambient conditions. The resulting residues were scraped off the slide with a steel razor blade and pressed between two sheets of weighing paper by hand to form sub-millimeter sized pellets. The prepared samples were mounted on polyimide single crystal sample loops. Xray diffraction data were measured on a Bruker X8 Prospector (Bruker AXS, Madison, WI, USA) single crystal diffractometer using $\mathrm{Cu}-\mathrm{K} \alpha$ radiation from a microfocus source with multilayer optics $(\lambda=1.54060 \AA$, power $=45 \mathrm{kV}, 0.60 \mathrm{~mA})$. A series of 15 4-minute-long photographs were taken while rotating the sample $360^{\circ}$ about the phi axis, giving a total irradiation time of one hour. X-ray intensities were recorded across the $2 \theta$ of $28.5^{\circ}$ to $60.5^{\circ}$ using an Apex II CCD area detector situated at a crystal-to-detector distance of $10.0 \mathrm{~cm}$ and with the detector remaining stationary throughout the measurement. Photographic data were reduced by radially integrating a $33^{\circ}$-wide sector of the photograph in shells of $0.02^{\circ}$ in $2 \theta$ using the Bruker Apex3 software suite ${ }^{115}$.

Synthesis of Mangiferin conjugated gold nanoparticles (MGF-AuNPs). The Mangiferin gold nanoparticles (MGF-AuNPs) were produced by mixing of $4.2 \mathrm{mg}$ mangiferin (MGF) in $6 \mathrm{~mL}$ of doubly deionized (DI) water. The solution was stirred at $100^{\circ} \mathrm{C}$ for $10 \mathrm{~min}$ to dissolve the MGF into water to get a clear solution. Sodium tetrachloroaurate $(100 \mu \mathrm{L}$ of $0.1 \mathrm{M})$ was added to the reaction mixture to produce gold nanoparticles (AuNPs). Change in color from yellow to burgundy wine red indicated the formation of MGF-AuNPs in the homogeneous reaction mixture. The MGF-AuNPs were characterized by various instrumentation techniques including, UV-Vis spectrophotometry ${ }^{116}$, Zetasizer Nano S90, TEM ${ }^{117}$ and ICP-MS ${ }^{118}$. For various in vitro and in vivo investigations, the treatment concentrations were calculated based on the amount of gold present in MGF-AuNPs. The amount of Au was calculated by ICP-MS technique.

In vitro stability analysis. The stability of MGF-AuNPs were confirmed by mixing gold nanoparticles with various biological solutions such as aqueous solutions of $1 \% \mathrm{NaCl}, 0.5 \%$ cysteine, $0.2 \mathrm{M}$ histidine, $0.5 \%$ human serum albumin (HSA), $0.5 \%$ bovine serum albumin (BSA), and $\mathrm{pH} 7$. The stability of the conjugates was measured by monitoring the surface plasmon resonance (SPR) ${ }^{119}$ at different time points for a week. A negligible/no change in SPR frequency confirmed stability and the retention of nanoparticulate composition in all mixtures. The MGF-AuNPs was also tested for their stability at different dilutions in the DI water using UV-visible spectrophotometry ${ }^{116}$. 
Cellular internalization and trafficking pathway. The endocytosis mode of MGF-AuNPs was investigated by pre-blocking various receptors onto PC-3 cells. The optimum dose and incubation time were determined by incubating PC-3 cells with various concentrations of Mangiferin conjugated gold nanoparticles (MGF-AuNPs) at different time points. Further, the cell trafficking pathway of MGF-AuNPs was evaluated by using various receptor blocking agents to confirm the clathrin and or caveolae mediated endocytosis. Chlorpromazine (CPZ) and X-22 anti-clathrin antibodies were chosen to inhibit clathrin-mediated endocytosis. AntiCaveolin-1 antibody was chosen to block/inhibit caveolae-mediated uptake. Internalization of MGF-Gold nanoparticles were monitored by two independent techniques: (i) Cytoviva dark field fluorescence microscopy and; (ii) transmission electron microscopy (TEM) ${ }^{117}$. For the dark field microscopic study, ultra clean and sterile cover slip was kept in 6 well plate to grow the PC-3 cells. For the study by TEM technique ${ }^{117}$, cells were grown on the plate without any coverslip.

Briefly, PC-3 cells $\left(10^{6} / \mathrm{mL}\right)$ were seeded into 6 well plates in RPMI medium and incubated for $24 \mathrm{~h}$ in $\mathrm{CO}_{2}$ incubator at $37^{\circ} \mathrm{C}$. The cells were pre-incubated with the inhibitors as follows: PBS (control), chlorpromazine hydrochloride $(10 \mu \mathrm{M} ; 20 \mathrm{~min}), \mathrm{X}-22$ anti-clathrin antibody

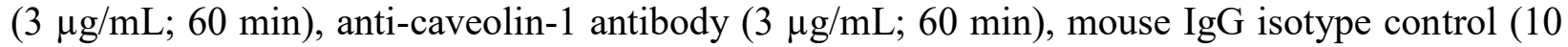
$\mu \mathrm{g} / \mathrm{mL} ; 60 \mathrm{~min})$, anti-fibronectin antibody $(3 \mu \mathrm{g} / \mathrm{mL} ; 60 \mathrm{~min})$, and laminin receptor antibody (ABLR) $(10 \mu \mathrm{g} / \mathrm{mL} ; 60 \mathrm{~min})$. The cells were incubated with all the inhibitors in $\mathrm{CO}_{2}$ incubator at $37^{\circ} \mathrm{C}$. Post incubation, cells were washed with $1 \mathrm{XPBS}$ twice followed by incubation with MGFAuNPs $(41 \mu \mathrm{M})$ for $60 \mathrm{~min}$ in the $\mathrm{CO}_{2}$ incubator at $37^{\circ} \mathrm{C}$. The samples were prepared by the following techniques:

Dark field microscopic technique. After incubation, cells were washed 10 times with 1XPBS, and fixed with $4 \%$ paraformaldehyde (PFA) for $10 \mathrm{~min}$ in the $\mathrm{CO}_{2}$ incubator at $37^{\circ} \mathrm{C}$. Cells were further washed 2 times with 1XPBS and slides were prepared by using DAPI nuclear dye and observed under CytoViva dark field microscope coupled with dual mode fluorescence. Cell morphology was initially observed, followed by the uptake of nanoparticles. Images were captured via Dage Imaging Software.

TEM technique. After incubation, cells were washed 10 times with 1XPBS, trypsinized and centrifuged into pellets, and fixed with $2 \%$ glutaraldehyde, $2 \%$ paraformaldehyde in sodium cacodylate buffer $(0.1 \mathrm{M})$. The cells were further fixed with $1 \%$ buffered osmium tetroxide in 2Mercaptoethanol buffer and dehydrated in graded acetone series and embedded in Epon-Spurr epoxy resin. Sections were cut at $85 \mathrm{~nm}$ using a diamond knife (Diatome, Hatfield PA). The sections were stained with Sato's triple lead stain and 5\% aqueous uranyl acetate for organelle visualization. The samples, as prepared above, were examined on JEOL 1400 TEM microscope (JEOL, Peabody, Mass.) operated at $80 \mathrm{kV}$ at the University of Missouri's Electron Microscopy Core Facility ${ }^{117}$.

MGF-AuNPs were explored for their selective affinity toward tumor cells by incubating with the same concentrations and time points as used for the normal endothelial cells (HAECs).

Cell viability assay. The effect of MGF-AuNPs on prostate cancer (PC-3) and normal human aortic endothelial (HAECs) cells viability was determined using MTT assay (Sigma). The intensity of developed color was measured by micro plate reader (Molecular device, USA) operating at $570 \mathrm{~nm}$ wavelength. Percent cell viability was calculated by using the formula: $(\mathrm{T} / \mathrm{C}) \times 100$, where $\mathrm{C}=$ Absorbance of control, $\mathrm{T}=$ Absorbance of treatment. The IC-50 values were calculated using the Origin software ${ }^{120}$. 
Apoptosis assay. PC-3 cells were incubated with MGF-AuNPs for $24 \mathrm{hr}$ and the experiment was performed according to the manufacture's protocol (FITC Annexin V Apoptosis Detection Kit I). The samples were analyzed by FACScan flow cytometry (FACSort, Becton Dickinson, USA). For each sample, 30,000 ungated events were acquired. ${ }^{121}$

Assessment of apoptotic and necrotic cell morphology. PC-3 cells were incubated with MGFAuNPs for $24 \mathrm{hr}$ and the experiment was performed according to the manufacture's protocol (GFP-CERTIFIED ${ }^{\circledR}$ Apoptosis/ Necrosis detection kit). The slides were prepared and visualized under fluorescent microscope with a dual filter set for Cyanine-3 (Ex/Em: 550/570 nm), 7-AAD (Ex/Em: 546/647) and GFP/FITC (Ex/Em: 488/514) (Olympus, USA).

In vitro anti-angiogenesis assay. In vitro anti-angiogenesis effect of MGF-AuNPs on HAECs cells was determined using tube formation assay. The test was performed according the manufacture's protocol (In vitro angiogenesis assay kit). Briefly, matrigel was coated in 96 well plate and the plates were incubated for $30 \mathrm{~min}$ at $37^{\circ} \mathrm{C}$. HAECs cells and test samples were added into the same plates and incubated for $24 \mathrm{hr}$ for tube formation analysis. The images were captured by fluoresce microscope, (Olympus, Center Valley, PA, USA) at 4x magnification after $24 \mathrm{hr}^{122}$.

NF-кB measurement. The Phospho-NF-кB p65 (Ser536) (93H1) Rabbit mAb (Alexa Fluor ${ }^{\circledR} 488$ Conjugate) Kit was used to study the effect of MGF-AuNPs on the expression of NF- $\kappa$ B. Briefly, PC-3 cells were seeded into 6 well plate at a density of $10^{6}$ cells $/ \mathrm{mL}$ and were incubated for $24 \mathrm{hr}$. The cells were treated with MGF-AuNPs $(40 \mu \mathrm{M})$ for $18 \mathrm{hr}$ and post-treated with TNF- $\alpha(0.1 \mathrm{nM})$ for another $30 \mathrm{~min}$ at $37^{\circ} \mathrm{C}$. The assay was performed as per kit instructions and the results were analyzed by FACScan flow cytometry (FACSort, Becton Dickinson, USA) with a minimum of 10,000 events being recorded.

Macrophage MGF-AuNPs uptake studies. RAW 264.7 macrophages were cultured in DMEM $+10 \%$ FBS in a $75 \mathrm{~cm}^{2}$ flask. The cells at a density of $10^{6}$ were plated in 6 well plates overnight for adherence. The cells were then replenished with fresh medium and incubated with MGFAuNPs $(40 \mu \mathrm{M})$ for 60 minutes. Cells were then analyzed for the presence of gold nanoparticles by CytoViva dark field microscopy.

The effect of MGF-AuNPs on NF-KB inhibition in RAW 264.7 macrophages. Cells were preincubated with MGF-AuNPs for $2 \mathrm{hr}$ followed by treatment with either LPS (positive control) or RANKL for 30 minutes. Cells were lysed and lysates were run on polyacrylamide gel electrophoresis and transferred onto nitrocellulose membrane. These membranes were then

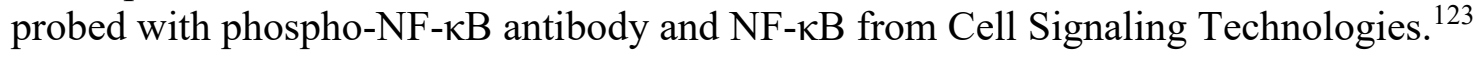

Cytokine analysis by real time PCR following treatment of RAW 264.7 cells with MGFAuNPs. RAW 264.7 cells were cultured in 6 well plates overnight for adherence followed by the treatment with MGF-AuNPs for $4 \mathrm{hr}$. LPS and Starch stabilized AuNPs (S-AuNPs) were used as positive and negative control respectively. The cells were lysed, and RNA was isolated using RNA isolation kit from Qiagen (Germantown, MD). RNA was then analyzed for IL-12, TNF- $\alpha$, IL-10 and IL-6 using real time PCR. ${ }^{124}$ 
Effect of MGF-AuNPs treated macrophages on prostate cancer cell proliferation. PC-3 cells at a density of $10^{5}$ cells/well were plated in 6 well plate overnight for adherence. Subsequently, RAW 264.7 macrophages were treated with MGF-AuNPs $(4 \mu \mathrm{M})$ for 18-24 hr. The macrophages were washed to remove unbound MGF-AuNPs in order to avoid the direct effect of MGF-AuNPs on cancer cells. In the meantime, the PC-3 cells were labelled with carboxyfluorescien succinimidyl ester (CFSE) to assess their proliferation. The macrophages were then co-cultured with PC-3 cells using a ratio of 10:1 (1 part of PC-3 cells to 10 part of macrophages) for $72 \mathrm{hr}$. The co-culture was observed under the fluorescent microscope and pictures taken. We observed significant reduction in the proliferation of PC-3 cells when cocultured with macrophages which were pre-treated with MGF-AuNPs ${ }^{125}$.

Animal studies. All in vivo work has been performed at an IACUC approved laboratory and in accordance with ARRIVE guidelines for animal welfare.

Ethics declarations: All experiments of MGF-AuNPs involving animals were approved by the Institutional Animal Care and Use Committees of the Harry S. Truman Memorial Veterans Hospital and the University of Missouri and were performed in accordance with the Guide for the Care and Use of Laboratory Animals under an IACUC approved protocol number 8767. Severe combined immuno-deficient SCID (ICR-SCID) male mice show a severe combined immunodeficiency (from Taconic Farms, Hudson, New York) were used for the therapeutic study. The mice used in our investigations weighed 24-27 g.

Description of animal procurement, housing, and grouping: Animals were maintained on a $12 \mathrm{~h}$ light-dark cycle and had access to sterilized standard chow and water ad libidum. Animals were allowed to acclimate for 7-10 days prior to initiation of work. Human prostate cancer cell line PC-3 was obtained from the American Type Culture Collection (ATCC; Manassas, VA), and cultured according to ATCC recommendations by the University of Missouri Cell and Immunobiology Core facility. Mice received ear tag identifiers under inhalational anesthesia (isoflurane/oxygen) followed by unilateral, subcutaneous hind flank inoculations of $10 \times 10^{6}$ PC3 cells suspended in $0.1 \mathrm{~mL}$ of sterile Dulbecco's phosphate buffered saline (DPBS) and Matrigel $^{\circledR}(2: 1, v: v)$. Solid tumors were allowed to develop over a period of 3 weeks, and animals were randomized (Day 0$)$ into control and treatment groups $(n=7)$ having no significant difference in tumor volumes $(p=0.64$; Student's t-test) or body weights $(p=0.17)$. Tumor volumes were estimated from caliper measurements using the formula $\mathrm{V}=$ length $\mathrm{x}$ width $\mathrm{x}$ depth. On Day 8, animals in the treatment group received intraperitoneal administrations of MGF-AuNP agent in DPBS $(100 \mu \mathrm{L})$ while under inhalational anesthesia in doses as outlined in the following section. Similarly, control animals received $30 \mu \mathrm{L}$ of saline intraperitoneally. No significant difference $(p=0.93)$ in tumor volume or body weight $(p=0.21)$ was noted between the groups. Tumor volumes, body weights and health status were then determined twice each week. At the end of the study (Day 42), mice were euthanized by cervical dislocation, and blood sample was collected by cardiac puncture. Samples of spleen, liver, tumor, and blood were harvested, weighed and submitted to the University of Missouri Neutron Activation Analysis (NAA) facility at the University of Missouri Research reactor (MURR) for the accurate quantification of gold in various tissues by NAA analysis.

NOTE: Although male mice have been selected in our investigations, it is important to note that this is a xenograft model, thus murine gender is not anticipated to significantly influence tumor 
biology. Importantly, as we are studying prostate cancer, the use of male mice only is appropriate.

In vivo bio-distribution study by Neutron Activation Analysis (NAA). To assess the gold content in various tissue in SCID mice $(\mathrm{n}=7) .1 .5 \mathrm{mg} / \mathrm{kg}$ bw of MGF-AuNPs were administered in these mice for seven weeks, while control mice $(n=7)$ did not receive any treatment with MGF-AuNPs. Tumor tissue, spleen, liver and blood were harvested upon euthanization, put into chloridometer sample vials and dried for approximately 48 hours at $100-120^{\circ} \mathrm{C}$. Dried tissue mass of approximately $0.5-1.0 \mathrm{~g}$ was placed into polyethylene vials (used for control of counting geometry). We estimated the amount of gold in various tissue samples as described previously through neutron activation analysis (NAA) techniques. ${ }^{38}$

In vivo therapeutic efficacy study. Antitumor efficacy of MGF-AuNPs was evaluated by using prostate tumors xenografts in SCID mice as developed above. Briefly, SCID male mice were subcutaneously inoculated with $10 \times 10^{6}$ PC-3 cells (suspended in $0.1 \mathrm{~mL}$ of sterile DPBS and Matrigel $\left.{ }^{\circledR}(2: 1, \mathrm{v}: \mathrm{v})\right)$ in the right hind flank under inhalation anesthesia (isoflurane/oxygen). After inoculation, tumors were allowed to grow for 2-3 weeks, at which time the tumors were measured by digital caliper measurements and calculated as length $\mathrm{x}$ width $\mathrm{x}$ height. The mice were randomly divided into four groups ( $\mathrm{n}=7 /$ group) with no significant difference in tumor volume, randomization was generated using the standard $=$ RAND() function in Microsoft Excel, and the day of randomization was considered the day zero of therapy study (Table 2). On day zero, mice were given intraperitoneal injections as follows: Group 1: saline treated $(100 \mu \mathrm{L})$; Group 2: MGF-AuNPs treated (0.5 mg/kg bw); Group 3: MGF-AuNPs treated (1.0 mg/kg bw) and Group 4: MGF-AuNPs treated $(1.5 \mathrm{mg} / \mathrm{kg}$ bw) - all in $100 \mu \mathrm{L}$ Dulbecco's PBS. Using this regimen, animals were treated twice per week until the end of the study (42 days). The animals were monitored for their tumor volume, body weight and health effects until they were sacrificed at the end of the study. The fifth group $(\mathrm{n}=7)$ was kept as control group (no tumor and no treatment) and served as a control for complete blood count (CBC) values and body weight measurements. Animals were sacrificed at the end of study. Measurement of tumor volumes were carried out twice each week until the end of the study (Day 42).

The tissues (spleen, liver, tumor tissue and blood) were isolated from prostate tumor xenografts and were submitted to the University of Missouri Neutron Activation Analysis (NAA) facility at the University of Missouri Research reactor (MURR) for the accurate quantification of gold in various tissues by NAA analysis.

Statistical analysis. All experimental data are described as mean \pm SEM. Statistical analysis was carried out using the one-way analysis of variances (ANOVA) using Graph Pad Prism software. $P<0.05$ was considered significant. 


\section{AUTHOR INFORMATION}

\section{Corresponding Author}

*To whom correspondence should be addressed.

Dr. Kattesh V Katti

Department of Radiology, Institute of Green Nanotechnology

University of Missouri, Columbia, MO, 65212, USA

E-mail: kattik@health.missouri.edu

\section{Author Contributions}

The scientific idea was conceptualized by Dr. KVK. Experimental design and related research work were performed by Dr. MK and Dr. AK. Analysis, interpretation of data and the overall manuscript was written through contributions of all authors. All authors have given approval to the final version of the manuscript.

\section{Funding Sources}

The research results reported in this manuscript was partially financed by Dhanvantari Nano Ayushadi Private Limited (DNA), Chennai, India. Logistical support from the University of Missouri, Department of Radiology and Institute of Green Nanotechnology are greatly acknowledged.

\section{Acknowledgements}

This project was partially supported by funds from Dhanvantari Nano Ayushadi (DNA), Private Limited, Chennai, India. Logistical support from the Institute of Green Nanotechnology, University of Missouri are gratefully acknowledged. We also thank Dr. Bettina Gentry Veterinary Pathobiology, University of Missouri, for collaborations related to histological studies. The authors also thank various in vitro and in vivo animal core facilities of the University of Missouri, Columbia.

\section{Competing interest}

The authors declare no competing interests. 
Figure legends:

Scheme 1: (A) Green Nanotechnology Motif of Mangiferin functionalized Gold Nanoparticles (MGF-AuNPs).

Figure 1. (A) UV-Vis spectra showing in vitro stability of MGF-AuNPs in aqueous solutions after 1-week of incubation. (B) Spectra illustrate that the absorbance intensity is linearly proportional to MGF-AuNPs concentrations.

Figure 2. Characterization of MGF-AuNPs by (A) UV-Visible spectra, (B) HR-TEM images, (C) Core size distribution histogram employing image J software (D) TEM images.

Note: TEM: transmission electron microscopy. HR-TEM: high resolution TEM.

Figure 3: Energy Dispersive Spectroscopy (EDS) analysis of MGF-AuNPs. Image (A) showing the presence of Au element, and image (B) mapping of Au element.

Figure 4: Powder X ray Diffraction (PXRD) pattern of evaporated residue for MGF-AuNPs.

Figure 5: Receptor mediated endocytosis of MGF-AuNPs. (A, D): Untreated PC-3 cells; (B, E): MGF-AuNPs $(41 \mu \mathrm{M})$ treated PC-3 cells; (C, F): Laminin receptors on PC-3 cells blocked with ABLR antibody and post treated with MGF-AuNPs, showing laminin receptor affinity of MGFAuNPs in PC-3 cells. Optical images by cytoviva dark field microscopy and microscopic images by TEM techniques.

Scheme 2: Mechanisms of endocytosis, cellular entry and cellular trafficking pathways of MGF-AuNPs into prostate tumor cells.

Figure 6: Clathrin mediated endocytosis of MGF-AuNPs (A-F): Dark field (CytoViva) microscopic images; (G-J): TEM Images showing PC-3 cells pretreated with Chlorpromazine, anticlarthrin $\mathrm{AB}$, anti-caveolae $\mathrm{AB}$, anti-fibronectin $\mathrm{AB}$, and mouse $\mathrm{IgG}$ Isotype control $\mathrm{AB}$, followed by treatment with MGF-AuNPs $(41 \mu \mathrm{M})$. Images infer clathrin dependent and caveolae independent pathways for the endocytosis of MGF-AuNPs in PC-3 cells.

Figure 7: Time dependent internalization of MGF-AuNPs $(41 \mu \mathrm{M})$ into PC-3 cells, images observed by TEM. (A) at $30 \mathrm{~min}$; (B-C) at $60 \mathrm{~min}$; (D-E) at $90 \mathrm{~min}$; (F) at $120 \mathrm{~min}$.

Figure 8: TEM images showing MGF-AuNPs uptake into human aortic endothelial cells (HAECs), 60 min post treatment of MGF-AuNPs $(41 \mu \mathrm{M})$. (A) HAECs cells control; no treatment; (B) MGF-AuNPs treated cells $(41 \mu \mathrm{M})$.

Figure 9: (A) In vitro therapeutic efficacy of MGF-AuNPs against prostate cancer cells (PC-3). (B) MGF-AuNPs showing non-toxic feature to normal cells (HAECs). Cells were treated using serial dilutions of MGF-AuNPs and incubated with MTT dye for cell viability measurements.

Figure 10: Apoptosis assays of MGF-AuNPs: (A) induction of apoptosis in PC-3 cells measured by Flow Cytometry; (B) Measured by Fluorescent Microscopy; (C) Inhibition of tube/capillary formation in HAECs endothelial cells confirming anti-angiogenesis effects. 
Figure 11: MGF-AuNPs target NF- $\kappa B$ as confirmed through inhibition of TNF- $\alpha$ stimulated NF$\kappa \mathrm{B}$ phosphorylation in PC-3 cells.

Figure 12: MGF-AuNPs induced polarization of macrophages. (A-D): RAW 264.7 cells were pretreated with either Starch-AuNPs (S-AuNPs as control), or MGF-AuNPs for $2 \mathrm{hr}$ and treated either with LPS $(100 \mathrm{ng} / \mathrm{mL})$ or RANKL $(25 \mathrm{ng} / \mathrm{ml})$ or left untreated for $4 \mathrm{hr}$. RNA was isolated from treated and untreated samples and analyzed for IL-12, TNF- $\alpha$, IL-10, and IL-6 by real time PCR using probes from TaqMan, Applied Biosystems. (E): RAW 264.7 cells were either treated with LPS (100 ng/mL) or Starch-AuNPs (S-AuNPs as control), or MGF-AuNPs or left untreated for 30 mins. The cells were lysed with 1X Lamellae buffer and lysates were run on PAGE gel and transferred onto nitrocellulose membranes. The membranes were than probed for either phospho- NF- $\kappa \mathrm{B}$ or NF- $\kappa \mathrm{B}$ using respective antibodies.

Scheme 3: Targeting ability of MGF-AuNPs toward tumor microenvironment (TME) and it's reprograming ability of M2 to M1 phenotype.

Figure 13: MGF-AuNPs uptake in (A) Raw 264.7 macrophages and (B) PC-3 tumor cells. The cells were post-treated with MGF-AuNPs $(40 \mu \mathrm{M})$ and images were taken using CytoViva dark field microscopy.

Figure 14: MGF-AuNPs-treated macrophages inhibited the proliferation of prostate tumor cells. Raw 264.7 macrophages were pre-treated with MGF-AuNPs. Separately, PC-3 cells were labelled with CFSE to assess their proliferation. The macrophages were then co-cultured with PC-3 cells for $72 \mathrm{hr}$. The images were obtained by fluorescent microscope.

Figure 15: Targeting of MGF-AuNP on splenic macrophages: (A) Biodistribution of MGF${ }^{198} \mathrm{AuNP}$ in normal mice showing selective uptake only in liver and spleen and limited/no uptake in non-target organs; (B) Biodistribution of MGF- ${ }^{198}$ AuNPs in prostate tumor bearing SCID mice. Gold concentrations in tumor, liver and spleen measured using neutron activation analysis (NAA) - showing limited uptake in tumors and major uptake in spleen and liver-suggesting targeting of MGF-AuNP on splenic macrophages.

Figure 16: In vivo therapeutic efficacy of MGF-AuNPs in SCID mice implanted with PC-3 prostate tumor cells ( $\mathrm{n}=7 / \mathrm{set})$. MGF-AuNPs were administered intraperitoneally. 


\section{References.}

1 Oudard, S. et al. Effect of Adding Docetaxel to Androgen-Deprivation Therapy in Patients With High-Risk Prostate Cancer With Rising Prostate-Specific Antigen Levels After Primary Local Therapy: A Randomized Clinical Trial. JAMA Oncol 5, 623-632, doi:10.1001/jamaoncol.2018.6607 (2019).

2 Nabid, A. et al. Duration of Androgen Deprivation Therapy in High-risk Prostate Cancer: A Randomized Phase III Trial. Eur Urol 74, 432-441, doi:10.1016/j.eururo.2018.06.018 (2018).

3 Shipley, W. U. et al. Radiation with or without Antiandrogen Therapy in Recurrent Prostate Cancer. N Engl J Med 376, 417-428, doi:10.1056/NEJMoa1607529 (2017).

4 Wallace, K., Landsteiner, A., Bunner, S., Engel-Nitz, N. \& Luckenbaugh, A. Epidemiology and mortality of metastatic castration-resistant prostate cancer (mCRPC) in a managed care population in the United States. Journal of Clinical Oncology 38, e13592-e13592, doi:10.1200/JCO.2020.38.15_suppl.e13592 (2020).

5 Sartor, O. \& de Bono, J. S. Metastatic Prostate Cancer. N Engl J Med 378, 645-657, doi:10.1056/NEJMra1701695 (2018).

6 Huang, Y., Jiang, X., Liang, X. \& Jiang, G. Molecular and cellular mechanisms of castration resistant prostate cancer. Oncol Lett 15, 6063-6076, doi:10.3892/ol.2018.8123 (2018).

7 Nuhn, P. et al. Update on Systemic Prostate Cancer Therapies: Management of Metastatic Castration-resistant Prostate Cancer in the Era of Precision Oncology. Eur Urol 75, 88-99, doi:10.1016/j.eururo.2018.03.028 (2019).

8 Messex, J. K., Byrd, C. J. \& Liou, G. Y. Signaling of Macrophages that Contours the Tumor Microenvironment for Promoting Cancer Development. Cells-Basel 9, doi:ARTN 91910.3390/cells9040919 (2020).

9 Di Mitri, D. et al. Re-education of Tumor-Associated Macrophages by CXCR2 Blockade Drives Senescence and Tumor Inhibition in Advanced Prostate Cancer. Cell Rep 28, 2156-+, doi:10.1016/j.celrep.2019.07.068 (2019).

10 Chen, Y. B. et al. Tumor-associated macrophages: an accomplice in solid tumor progression. J Biomed Sci 26, doi:ARTN 7810.1186/s12929-019-0568-z (2019).

11 Sanaei, M. J., Salimzadeh, L. \& Bagheri, N. Crosstalk between myeloid-derived suppressor cells and the immune system in prostate cancer: MDSCs and immune system in Prostate cancer. J Leukocyte Biol 107, 43-56, doi:10.1002/Jlb.4ru0819-150rr (2020).

12 Sivagnanalingam, U., Beatty, P. L. \& Finn, O. J. Myeloid derived suppressor cells in cancer, premalignancy and inflammation: A roadmap to cancer immunoprevention. $\mathrm{Mol}$ Carcinogen 59, 852-861, doi:10.1002/mc.23206 (2020).

$13 \mathrm{Lu}, \mathrm{X}$. et al. Effective combinatorial immunotherapy for castration-resistant prostate cancer. Nature 543, 728-732, doi:10.1038/nature21676 (2017).

14 Calcinotto, A. et al. IL-23 secreted by myeloid cells drives castration-resistant prostate cancer. Nature 559, 363-+, doi:10.1038/s41586-018-0266-0 (2018).

15 Jeannin, P., Paolini, L., Adam, C. \& Delneste, Y. The roles of CSFs on the functional polarization of tumor-associated macrophages. FEBS $J$ 285, 680-699, doi:10.1111/febs.14343 (2018).

16 Guerriero, J. L. Macrophages: The Road Less Traveled, Changing Anticancer Therapy. Trends Mol Med 24, 472-489, doi:10.1016/j.molmed.2018.03.006 (2018). 
17 Dang, T. \& Liou, G. Y. Macrophage Cytokines Enhance Cell Proliferation of Normal Prostate Epithelial Cells through Activation of ERK and Akt. Sci Rep-Uk 8, doi:ARTN 771810.1038/s41598-018-26143-8 (2018).

18 Lanciotti, M. et al. The Role of M1 and M2 Macrophages in Prostate Cancer in relation to Extracapsular Tumor Extension and Biochemical Recurrence after Radical Prostatectomy. Biomed Res Int 2014, doi:Artn 48679810.1155/2014/486798 (2014).

19 Verzella, D. et al. Life, death, and autophagy in cancer: NF-kappa B turns up everywhere. Cell Death Dis 11, doi:ARTN 21010.1038/s41419-020-2399-y (2020).

20 Karin, M. \& Lin, A. NF-kappa B at the crossroads of life and death. Nat Immunol 3, 221227, doi:10.1038/ni0302-221 (2002).

21 Verzella, D. et al. Targeting the NF-kappaB pathway in prostate cancer: a promising therapeutic approach? Curr Drug Targets 17, 311-320, doi:10.2174/1389450116666150907100715 (2016).

22 Staal, J. \& Beyaert, R. Inflammation and NF-kappaB Signaling in Prostate Cancer: Mechanisms and Clinical Implications. Cells-Basel 7, doi:10.3390/cells7090122 (2018).

23 Nguyen, D. P., Li, J. Y., Yadav, S. S. \& Tewari, A. K. Recent insights into NF-kappa B signalling pathways and the link between inflammation and prostate cancer. Вju Int 114, 168-176, doi:10.1111/bju.12488 (2014).

24 Rotimi, S. O. et al. Gene Expression Profiling Analysis Reveals Putative Phytochemotherapeutic Target for Castration-Resistant Prostate Cancer. Front Oncol 9, 714, doi:10.3389/fonc.2019.00714 (2019).

25 Salehi, B. et al. Phytochemicals in Prostate Cancer: From Bioactive Molecules to Upcoming Therapeutic Agents. Nutrients 11, doi:10.3390/nu11071483 (2019).

26 Seca, A. M. L. \& Pinto, D. C. G. A. Plant Secondary Metabolites as Anticancer Agents: Successes in Clinical Trials and Therapeutic Application. Int J Mol Sci 19, doi:ARTN 26310.3390/ijms19010263 (2018).

27 Shokoohinia, Y. et al. Potential Anticancer Properties of Osthol: A Comprehensive Mechanistic Review. Nutrients 10, doi:ARTN 3610.3390/nu10010036 (2018).

28 Sharma, P., McClees, S. F. \& Afaq, F. Pomegranate for Prevention and Treatment of Cancer: An Update. Molecules 22, doi:10.3390/molecules22010177 (2017).

29 Casey, S. C. et al. Cancer prevention and therapy through the modulation of the tumor microenvironment. Semin Cancer Biol 35, S199-S223, doi:10.1016/j.semcancer.2015.02.007 (2015).

30 Cragg, G. M. \& Newman, D. J. Natural products: A continuing source of novel drug leads. Bba-Gen Subjects 1830, 3670-3695, doi:10.1016/j.bbagen.2013.02.008 (2013).

31 Zulkipli, I. N., David, S. R., Rajabalaya, R. \& Idris, A. Medicinal Plants: A Potential Source of Compounds for Targeting Cell Division. Drug Target Insight 9, 9-19, doi:10.4137/Dti.S24946 (2015).

32 Weaver, B. A. How Taxol/paclitaxel kills cancer cells. Mol Biol Cell 25, 2677-2681, doi:10.1091/mbc.E14-04-0916 (2014).

33 Shukla, R. et al. Laminin receptor specific therapeutic gold nanoparticles ((AuNP)-Au198-EGCg) show efficacy in treating prostate cancer. P Natl Acad Sci USA 109, 1242612431, doi:10.1073/pnas.1121174109 (2012). 
34 Chanda, N. et al. Bombesin functionalized gold nanoparticles show in vitro and in vivo cancer receptor specificity. $P$ Natl Acad Sci USA 107, 8760-8765, doi:10.1073/pnas.1002143107 (2010).

35 Khoobchandani, M. et al. New Approaches in Breast Cancer Therapy Through Green Nanotechnology and Nano-Ayurvedic Medicine - Pre-Clinical and Pilot Human Clinical Investigations. Int J Nanomed 15, 181-197, doi:10.2147/Ijn.S219042 (2020).

36 Thipe, V. C. et al. Development of resveratrol-conjugated gold nanoparticles: interrelationship of increased resveratrol corona on anti-tumor efficacy against breast, pancreatic and prostate cancers. Int J Nanomed 14, 4413-4428, doi:10.2147/Ijn.S204443 (2019).

37 Khoobchandani M, Katti. K., Karikachery RA, Thipe CV, Bloebaum LRP, Katti VK. in Biotechnology Products in Everyday Life (ed Menka KhoobchandaniArpita Saxena) 3752 (Springer 2019).

38 Katti, K. V. et al. Prostate tumor therapy advances in nuclear medicine: green nanotechnology toward the design of tumor specific radioactive gold nanoparticles. $J$ Radioanal Nucl Ch 318, 1737-1747, doi:10.1007/s10967-018-6320-4 (2018).

39 Al-Yasiri, A. Y. et al. Mangiferin functionalized radioactive gold nanoparticles (MGF(198)AuNPs) in prostate tumor therapy: green nanotechnology for production, in vivo tumor retention and evaluation of therapeutic efficacy. Dalton T 46, 14561-14571, doi:10.1039/c7dt00383h (2017).

40 Khoobchandani M, Zambre. A., Katti KK, Lin HC, Katti VK. Green nanotechnology from brassicaceae: development of broccoli phytochemicals-encapsulated gold nanoparticles and their applications in nanomedicine. International Journal of Green Nanotechnology 1, doi:doi.org/10.1177/1943089213509474 (2013).

41 Nune, S. K. et al. Green nanotechnology from tea: phytochemicals in tea as building blocks for production of biocompatible gold nanoparticles. J Mater Chem 19, 2912-2920, doi:10.1039/b822015h (2009).

42 Shukla, R. et al. Soybeans as a phytochemical reservoir for the production and stabilization of biocompatible gold nanoparticles. Small 4, 1425-1436, doi:10.1002/smll.200800525 (2008).

43 Katti, K. et al. Green Nanotechnology from Cumin Phytochemicals: Generation of Biocompatible Gold Nanoparticles. Int J Green Nanotechnol Biomed 1, B39-B52, doi:10.1080/19430850902931599 (2009).

44 Kattumuri, V. et al. Gum arabic as a phytochemical construct for the stabilization of gold nanoparticles: In vivo pharmacokinetics and X-ray-contrast-imaging studies. Small 3, 333-341, doi:10.1002/smll.200600427 (2007).

45 Chanda, N. et al. Radioactive gold nanoparticles in cancer therapy: therapeutic efficacy studies of GA-(AuNP)-Au-198 nanoconstruct in prostate tumor-bearing mice. NanomedNanotechnol 6, 201-209, doi:10.1016/j.nano.2009.11.001 (2010).

46 Karra, S. R. et al. Tc-99m-labeling and in vivo studies of a bombesin analogue with a novel water-soluble dithiadiphosphine-based bifunctional chelating agent. Bioconjugate Chem 10, 254-260, doi:DOI 10.1021/bc980096a (1999).

47 Chanda, N., Shukla, R., Katti, K. V. \& Kannan, R. Gastrin releasing protein receptor specific gold nanorods: breast and prostate tumor avid nanovectors for molecular imaging. Nano Lett 9, 1798-1805, doi:10.1021/n18037147 (2009). 
48 Kannan, R. et al. Nanocompatible chemistry toward fabrication of target-specific gold nanoparticles. J Am Chem Soc 128, 11342-11343, doi:10.1021/ja063280c (2006).

49 Chanda, N. et al. An Effective Strategy for the Synthesis of Biocompatible Gold Nanoparticles Using Cinnamon Phytochemicals for Phantom CT Imaging and Photoacoustic Detection of Cancerous Cells. Pharm Res-Dordr 28, 279-291, doi:10.1007/s11095-010-0276-6 (2011).

50 Fent, G. M. et al. Biodistribution of maltose and gum arabic hybrid gold nanoparticles after intravenous injection in juvenile swine. Nanomedicine 5, 128-135, doi:10.1016/j.nano.2009.01.007 (2009).

51 Pillarsetty, N. et al. In vitro and in vivo antitumor properties of tetrakis((trishydroxymethyl)phosphine)gold(I) chloride. J Med Chem 46, 1130-1132, doi:10.1021/jm025615g (2003).

52 Masibo, M. \& He, Q. Major mango polyphenols and their potential significance to human health. Compr Rev Food Sci F 7, 309-319, doi:10.1111/j.1541-4337.2008.00047.x (2008).

53 Dar, A. et al. Analgesic and antioxidant activity of mangiferin and its derivatives: the structure activity relationship. Biol Pharm Bull 28, 596-600, doi:DOI 10.1248/bpb.28.596 (2005).

54 Du, S. Y. et al. Mangiferin: An effective therapeutic agent against several disorders. Mol Med Rep 18, 4775-4786, doi:10.3892/mmr.2018.9529 (2018).

55 Guha, S., Ghosal, S. \& Chattopadhyay, U. Antitumor, immunomodulatory and anti-HIV effect of mangiferin, a naturally occurring glucosylxanthone. Chemotherapy 42, 443-451, doi:Doi 10.1159/000239478 (1996).

56 Imran, M. et al. Mangiferin: a natural miracle bioactive compound against lifestyle related disorders. Lipids Health Dis 16, doi:ARTN 8410.1186/s12944-017-0449-y (2017).

57 Li, H. Z. et al. Mangiferin exerts antitumor activity in breast cancer cells by regulating matrix metalloproteinases, epithelial to mesenchymal transition, and beta-catenin signaling pathway. Toxicol Appl Pharm 272, 180-190, doi:10.1016/j.taap.2013.05.011 (2013).

58 Shi, Z. L. et al. In Vitro and In Vivo Effects of Norathyriol and Mangiferin on alphaGlucosidase. Biochem Res Int 2017, doi:Artn 120601510.1155/2017/1206015 (2017).

59 Rajendran, P., Rengarajan, T., Nandakumar, N., Divya, H. \& Nishigaki, I. Mangiferin in cancer chemoprevention and treatment: pharmacokinetics and molecular targets. J Recept Sig Transd 35, 76-84, doi:10.3109/10799893.2014.931431 (2015).

60 Takeda, T. et al. Mangiferin, a novel nuclear factor kappa B-inducing kinase inhibitor, suppresses metastasis and tumor growth in a mouse metastatic melanoma model. Toxicol Appl Pharm 306, 105-112, doi:10.1016/j.taap.2016.07.005 (2016).

61 Tan, H. Y. et al. Repression of WT1-Mediated LEF1 Transcription by Mangiferin Governs beta-Catenin-Independent Wnt Signalling Inactivation in Hepatocellular Carcinoma. Cell Physiol Biochem 47, 1819-1834, doi:10.1159/000491063 (2018).

62 Sanchez, G. M. et al. Protective effects of Mangifera indica L. extract, mangiferin and selected antioxidants against TPA-induced biomolecules oxidation and peritoneal macrophage activation in mice. Pharmacol Res 42, 565-573, doi:DOI 10.1006/phrs.2000.0727 (2000). 
63 Ramirez, N. M. et al. Extraction of Mangiferin and Chemical Characterization and Sensorial Analysis of Teas from Mangifera indica L. Leaves of the Uba Variety. Beverages 2, doi:ARTN 3310.3390/beverages2040033 (2016).

64 Lauricella, M., Emanuele, S., Calvaruso, G., Giuliano, M. \& D'Anneo, A. Multifaceted Health Benefits of Mangifera indica L. (Mango): The Inestimable Value of Orchards Recently Planted in Sicilian Rural Areas. Nutrients 9, doi:ARTN 52510.3390/nu9050525 (2017).

65 da Veiga Moreira, J. et al. Metabolic therapies inhibit tumor growth in vivo and in silico. Sci Rep 9, 3153, doi:10.1038/s41598-019-39109-1 (2019).

66 Israel, M. \& Schwartz, L. The metabolic advantage of tumor cells. Mol Cancer 10, 70, doi:10.1186/1476-4598-10-70 (2011).

67 Rousselle, P. \& Scoazec, J. Y. Laminin 332 in cancer: When the extracellular matrix turns signals from cell anchorage to cell movement. Semin Cancer Biol 62, 149-165, doi:10.1016/j.semcancer.2019.09.026 (2020).

68 Umbaugh, C. S., Diaz-Quinones, A., Neto, M. F., Shearer, J. J. \& Figueiredo, M. L. A dock derived compound against laminin receptor (37 LR) exhibits anti-cancer properties in a prostate cancer cell line model. Oncotarget 9, 5958-5978, doi:10.18632/oncotarget.23236 (2018).

69 Elkin, S. R., Lakoduk, A. M. \& Schmid, S. L. Endocytic pathways and endosomal trafficking: a primer. Wien Med Wochenschr 166, 196-204, doi:10.1007/s10354-0160432-7 (2016).

70 Satcher, R. L. et al. Cadherin-11 endocytosis through binding to clathrin promotes cadherin-11-mediated migration in prostate cancer cells. Journal of Cell Science 128, 4629-4641, doi:10.1242/jcs.176081 (2015).

$71 \mathrm{Fu}, \mathrm{P}$. et al. The different functions and clinical significances of caveolin-1 in human adenocarcinoma and squamous cell carcinoma. Oncotargets Ther 10, 819-835, doi:10.2147/Ott.S123912 (2017).

$72 \mathrm{Li}, \mathrm{L}$. K. et al. Caveolin-1 mediates testosterone-stimulated survival/clonal growth and promotes metastatic activities in prostate cancer cells. Cancer Res 61, 4386-4392 (2001).

73 DiGiacomo, V. \& Meruelo, D. Looking into laminin receptor: critical discussion regarding the non-integrin 37/67-kDa laminin receptor/RPSA protein. Biol Rev 91, 288310, doi:10.1111/brv.12170 (2016).

74 Lugano, R., Ramachandran, M. \& Dimberg, A. Tumor angiogenesis: causes, consequences, challenges and opportunities. Cell Mol Life Sci 77, 1745-1770, doi:10.1007/s00018-019-03351-7 (2020).

75 Ziyad, S. \& Iruela-Arispe, M. L. Molecular mechanisms of tumor angiogenesis. Genes Cancer 2, 1085-1096, doi:10.1177/1947601911432334 (2011).

76 Lugano, R., Ramachandran, M. \& Dimberg, A. Tumor angiogenesis: causes, consequences, challenges and opportunities. Cell Mol Life Sci 77, 1745-1770, doi:10.1007/s00018-019-03351-7 (2020).

77 Chagas, C. M. \& Alisaraie, L. Metabolites of Vinca Alkaloid Vinblastine: Tubulin Binding and Activation of Nausea-Associated Receptors. ACS Omega 4, 9784-9799, doi:10.1021/acsomega.9b00652 (2019). 
78 Klement, G. et al. Continuous low-dose therapy with vinblastine and VEGF receptor-2 antibody induces sustained tumor regression without overt toxicity (vol 105, 15, 2000). $J$ Clin Invest 116, 2827-2827, doi:10.1172/Jci08829c1 (2006).

79 Chen, L. et al. A NF-kB-Activin A signaling axis enhances prostate cancer metastasis. Oncogene 39, 1634-1651, doi:10.1038/s41388-019-1103-0 (2020).

80 Lessard, L. et al. NF-kappa B nuclear localization and its prognostic significance in prostate cancer. BJU Int 91, 417-420, doi:10.1046/j.1464-410x.2003.04104.x (2003).

81 Fares, J., Fares, M. Y., Khachfe, H. H., Salhab, H. A. \& Fares, Y. Molecular principles of metastasis: a hallmark of cancer revisited. Signal Transduct Target Ther 5, 28, doi:10.1038/s41392-020-0134-x (2020).

$82 \mathrm{Ji}, \mathrm{Z} ., \mathrm{He}, \mathrm{L} ., \mathrm{Regev}, \mathrm{A}$. \& Struhl, K. Inflammatory regulatory network mediated by the joint action of NF-kB, STAT3, and AP-1 factors is involved in many human cancers. Proc Natl Acad Sci U S A 116, 9453-9462, doi:10.1073/pnas.1821068116 (2019).

83 Pikarsky, E. et al. NF-kappaB functions as a tumour promoter in inflammation-associated cancer. Nature 431, 461-466, doi:10.1038/nature02924 (2004).

84 Guo, Q. et al. New Mechanisms of Tumor-Associated Macrophages on Promoting Tumor Progression: Recent Research Advances and Potential Targets for Tumor Immunotherapy. J Immunol Res 2016, 9720912, doi:10.1155/2016/9720912 (2016).

85 Annibaldi, A. \& Meier, P. Checkpoints in TNF-Induced Cell Death: Implications in Inflammation and Cancer. Trends Mol Med 24, 49-65, doi:10.1016/j.molmed.2017.11.002 (2018).

86 Hop, H. T. et al. Activation of NF-kB-Mediated TNF-Induced Antimicrobial Immunity Is Required for the Efficient Brucella abortus Clearance in RAW 264.7 Cells. Front Cell Infect Microbiol 7, 437, doi:10.3389/fcimb.2017.00437 (2017).

87 Bras, J. P. et al. TNF-alpha-induced microglia activation requires miR-342: impact on NF-kB signaling and neurotoxicity. Cell Death Dis 11, 415, doi:10.1038/s41419-0202626-6 (2020).

88 Mahdavi Sharif, P., Jabbari, P., Razi, S., Keshavarz-Fathi, M. \& Rezaei, N. Importance of TNF-alpha and its alterations in the development of cancers. Cytokine 130, 155066, doi:10.1016/j.cyto.2020.155066 (2020).

89 Bao, B. et al. The immunological contribution of NF-kappaB within the tumor microenvironment: a potential protective role of zinc as an anti-tumor agent. Biochim Biophys Acta 1825, 160-172, doi:10.1016/j.bbcan.2011.11.002 (2012).

90 Bercovici, N., Guerin, M. V., Trautmann, A. \& Donnadieu, E. The Remarkable Plasticity of Macrophages: A Chance to Fight Cancer. Front Immunol 10, 1563, doi:10.3389/fimmu.2019.01563 (2019).

91 Almatroodi, S. A., McDonald, C. F., Darby, I. A. \& Pouniotis, D. S. Characterization of M1/M2 Tumour-Associated Macrophages (TAMs) and Th1/Th2 Cytokine Profiles in Patients with NSCLC. Cancer Microenviron 9, 1-11, doi:10.1007/s12307-015-0174-x (2016).

92 Goerdt, S. et al. Alternative versus classical activation of macrophages. Pathobiology 67, 222-226, doi:10.1159/000028096 (1999).

93 Zhang, Q. et al. Tumor infiltrating M2 macrophages could predict biochemical recurrence of localized prostate cancer after radical prostatectomy. Exp Cell Res 384, 111588, doi:10.1016/j.yexcr.2019.111588 (2019). 
94 Yuri, P. et al. Increased tumor-associated macrophages in the prostate cancer microenvironment predicted patients' survival and responses to androgen deprivation therapies in Indonesian patients cohort. Prostate Int 8, 62-69, doi:10.1016/j.prnil.2019.12.001 (2020).

95 Werneck-Gomes, H. et al. Tumor-Associated Macrophages (TAM) are recruited to the aging prostate epithelial lesions and become intermingled with basal cells. Andrology 8, 1375-1386, doi:10.1111/andr.12783 (2020).

96 Guan, H. et al. Tumor-associated macrophages promote prostate cancer progression via exosome-mediated miR-95 transfer. J Cell Physiol, doi:10.1002/jcp.29784 (2020).

97 Zhang, F. et al. Genetic programming of macrophages to perform anti-tumor functions using targeted mRNA nanocarriers. Nat Commun 10, doi:ARTN 397410.1038/s41467019-11911-5 (2019).

98 Mei, J., Zhou, W. J., Li, S. Y., Li, M. Q. \& Sun, H. X. Interleukin-22 secreted by ectopic endometrial stromal cells and natural killer cells promotes the recruitment of macrophages through promoting CCL2 secretion. Am J Reprod Immunol 82, doi:ARTN e1316610.1111/aji.13166 (2019).

99 Muraoka, D. et al. Antigen delivery targeted to tumor-associated macrophages overcomes tumor immune resistance. $J$ Clin Invest 129, 1278-1294, doi:10.1172/JCI97642 (2019).

100 Zhao, X. et al. Trichomicin Suppresses Colorectal Cancer via Comprehensive Regulation of IL-6 and TNFalpha in Tumor Cells, TAMs, and CAFs. Front Pharmacol 11, 386, doi:10.3389/fphar.2020.00386 (2020).

101 Panni, R. Z., Linehan, D. C. \& DeNardo, D. G. Targeting tumor-infiltrating macrophages to combat cancer. Immunotherapy-Uk 5, 1075-1087, doi:10.2217/imt.13.102 (2013).

102 Bussard, K. M., Mutkus, L., Stumpf, K., Gomez-Manzano, C. \& Marini, F. C. Tumorassociated stromal cells as key contributors to the tumor microenvironment. Breast Cancer Res 18, doi:ARTN 8410.1186/s13058-016-0740-2 (2016).

103 Kumar, V., Patel, S., Tcyganov, E. \& Gabrilovich, D. I. The Nature of Myeloid-Derived Suppressor Cells in the Tumor Microenvironment. Trends Immunol 37, 208-220, doi:10.1016/j.it.2016.01.004 (2016).

104 Ferlazzo, G. et al. The abundant NK cells in human secondary lymphoid tissues require activation to express killer cell Ig-like receptors and become cytolytic. J Immunol 172, 1455-1462, doi:DOI 10.4049/jimmunol.172.3.1455 (2004).

105 Bronte, V. \& Pittet, M. J. The Spleen in Local and Systemic Regulation of Immunity. Immunity 39, 806-818, doi:10.1016/j.immuni.2013.10.010 (2013).

106 Lewis, S. M., Williams, A. \& Eisenbarth, S. C. Structure and function of the immune system in the spleen. Sci Immunol 4, doi:10.1126/sciimmunol.aau6085 (2019).

107 Suzuki, E., Kapoor, V., Jassar, A. S., Kaiser, L. R. \& Albelda, S. M. Gemcitabine selectively eliminates splenic Gr-1+/CD11b+ myeloid suppressor cells in tumor-bearing animals and enhances antitumor immune activity. Clin Cancer Res 11, 6713-6721, doi:10.1158/1078-0432.CCR-05-0883 (2005).

108 Germano, G. et al. Role of macrophage targeting in the antitumor activity of trabectedin. Cancer Cell 23, 249-262, doi:10.1016/j.ccr.2013.01.008 (2013). 
109 Colino, C. I., Lanao, J. M. \& Gutierrez-Millan, C. Targeting of Hepatic Macrophages by Therapeutic Nanoparticles. Frontiers in Immunology 11, doi:ARTN 21810.3389/fimmu.2020.00218 (2020).

110 Vallance, B. A., Deng, W., Knodler, L. A. \& Finlay, B. B. Mice lacking T and B lymphocytes develop transient colitis and crypt hyperplasia yet suffer impaired bacterial clearance during Citrobacter rodentium infection. Infect Immun 70, 2070-2081, doi:10.1128/iai.70.4.2070-2081.2002 (2002).

111 Ngo, M. T. \& Harley, B. A. C. Angiogenic biomaterials to promote therapeutic regeneration and investigate disease progression. Biomaterials 255, 120207, doi:10.1016/j.biomaterials.2020.120207 (2020).

112 Qin, S. et al. Recent advances on anti-angiogenesis receptor tyrosine kinase inhibitors in cancer therapy. J Hematol Oncol 12, 27, doi:10.1186/s13045-019-0718-5 (2019).

113 Inai, T. et al. Inhibition of vascular endothelial growth factor (VEGF) signaling in cancer causes loss of endothelial fenestrations, regression of tumor vessels, and appearance of basement membrane ghosts. Am $J$ Pathol 165, 35-52, doi:10.1016/S00029440(10)63273-7 (2004).

114 Heine, A., Held, S. A., Bringmann, A., Holderried, T. A. \& Brossart, P. Immunomodulatory effects of anti-angiogenic drugs. Leukemia 25, 899-905, doi:10.1038/leu.2011.24 (2011).

115 Techane, S. D., Gamble, L. J. \& Castner, D. G. X-ray photoelectron spectroscopy characterization of gold nanoparticles functionalized with amine-terminated alkanethiols. Biointerphases 6, 98, doi:10.1116/1.3622481 (2011).

116 Gajendiran, M., Jainuddin Yousuf, S. M., Elangovan, V. \& Balasubramanian, S. Gold nanoparticle conjugated PLGA-PEG-SA-PEG-PLGA multiblock copolymer nanoparticles: synthesis, characterization, in vivo release of rifampicin. J Mater Chem B 2, 418-427, doi:10.1039/c3tb21113d (2014).

117 Brun, F., Travan, A., Accardo, A. \& Paoletti, S. Characterization of silver nanoparticles for biomedical applications by means of quantitative analysis of tem micrographs biomed 2010. Biomed Sci Instrum 46, 105-110 (2010).

118 Scheffer, A., Engelhard, C., Sperling, M. \& Buscher, W. ICP-MS as a new tool for the determination of gold nanoparticles in bioanalytical applications. Anal Bioanal Chem 390, 249-252, doi:10.1007/s00216-007-1576-5 (2008).

119 Couture, M., Zhao, S. S. \& Masson, J. F. Modern surface plasmon resonance for bioanalytics and biophysics. Phys Chem Chem Phys 15, 11190-11216, doi:10.1039/c3cp50281c (2013).

120 Sanchez-Valdeolivar, C. A. et al. Phytochemical profile and antiproliferative effect of Ficus crocata extracts on triple-negative breast cancer cells. BMC Complement Med Ther 20, 191, doi:10.1186/s12906-020-02993-6 (2020).

121 Vermes, I., Haanen, C., Steffens-Nakken, H. \& Reutelingsperger, C. A novel assay for apoptosis. Flow cytometric detection of phosphatidylserine expression on early apoptotic cells using fluorescein labelled Annexin V. J Immunol Methods 184, 39-51, doi:10.1016/0022-1759(95)00072-i (1995).

122 Xie, X. \& Percipalle, P. Elevated transforming growth factor beta signaling activation in beta-actin-knockout mouse embryonic fibroblasts enhances myofibroblast features. $J$ Cell Physiol 233, 8884-8895, doi:10.1002/jcp.26808 (2018). 
123 Yang, F., Tang, E., Guan, K. \& Wang, C. Y. IKK beta plays an essential role in the phosphorylation of RelA/p65 on serine 536 induced by lipopolysaccharide. J Immunol 170, 5630-5635 (2003).

124 Sene, A. et al. Impaired cholesterol efflux in senescent macrophages promotes agerelated macular degeneration. Cell Metab 17, 549-561, doi:10.1016/j.cmet.2013.03.009 (2013).

125 Yan, C. et al. Visualizing Engrafted Human Cancer and Therapy Responses in Immunodeficient Zebrafish. Cell 177, 1903-1914 e1914, doi:10.1016/j.cell.2019.04.004 (2019). 
Figures

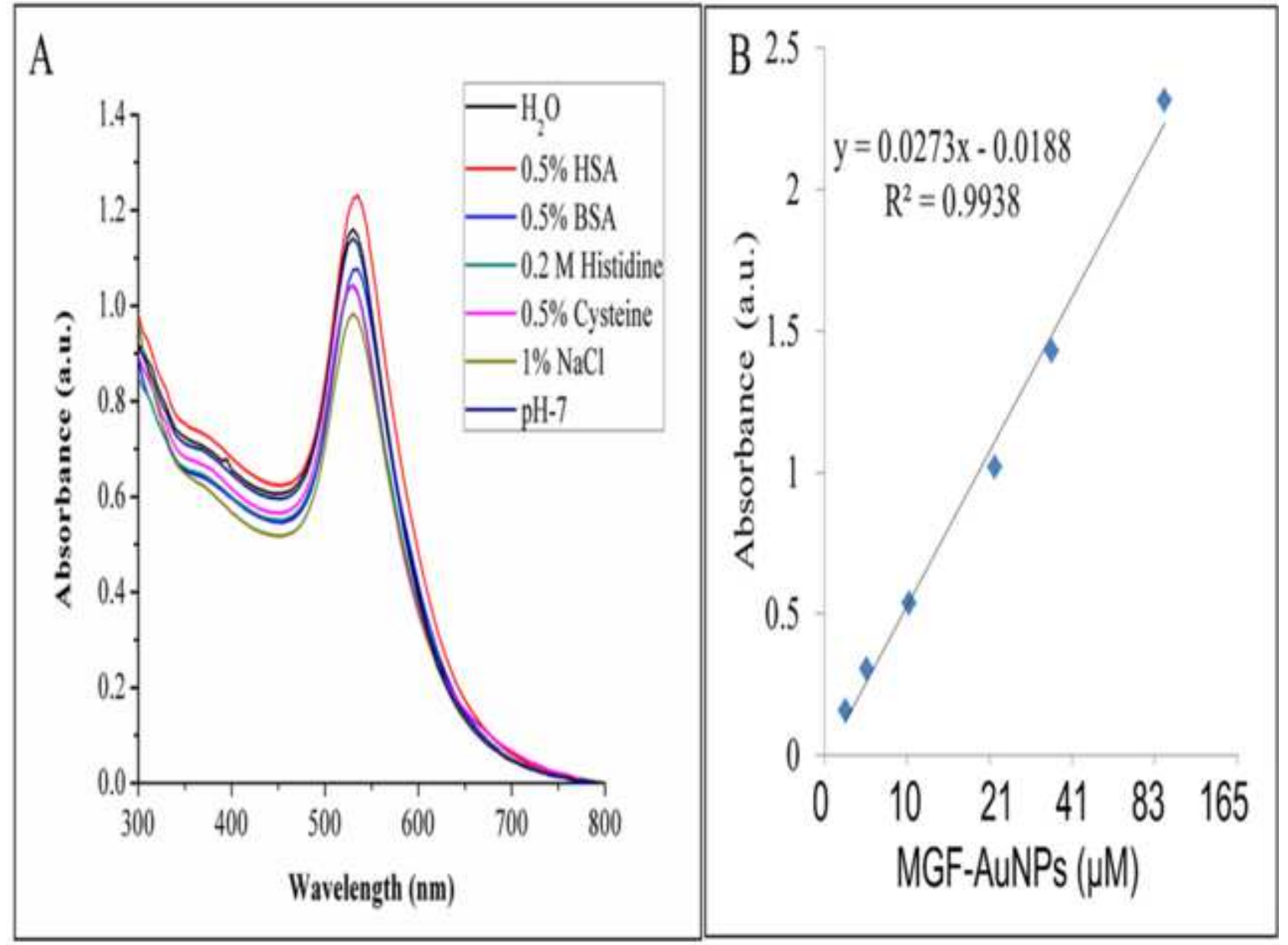

Figure 1

(A) UV-Vis spectra showing in vitro stability of MGF-AuNPs in aqueous solutions after 1-week of incubation. (B) Spectra illustrate that the absorbance intensity is linearly proportional to MGF-AuNPs concentrations. 

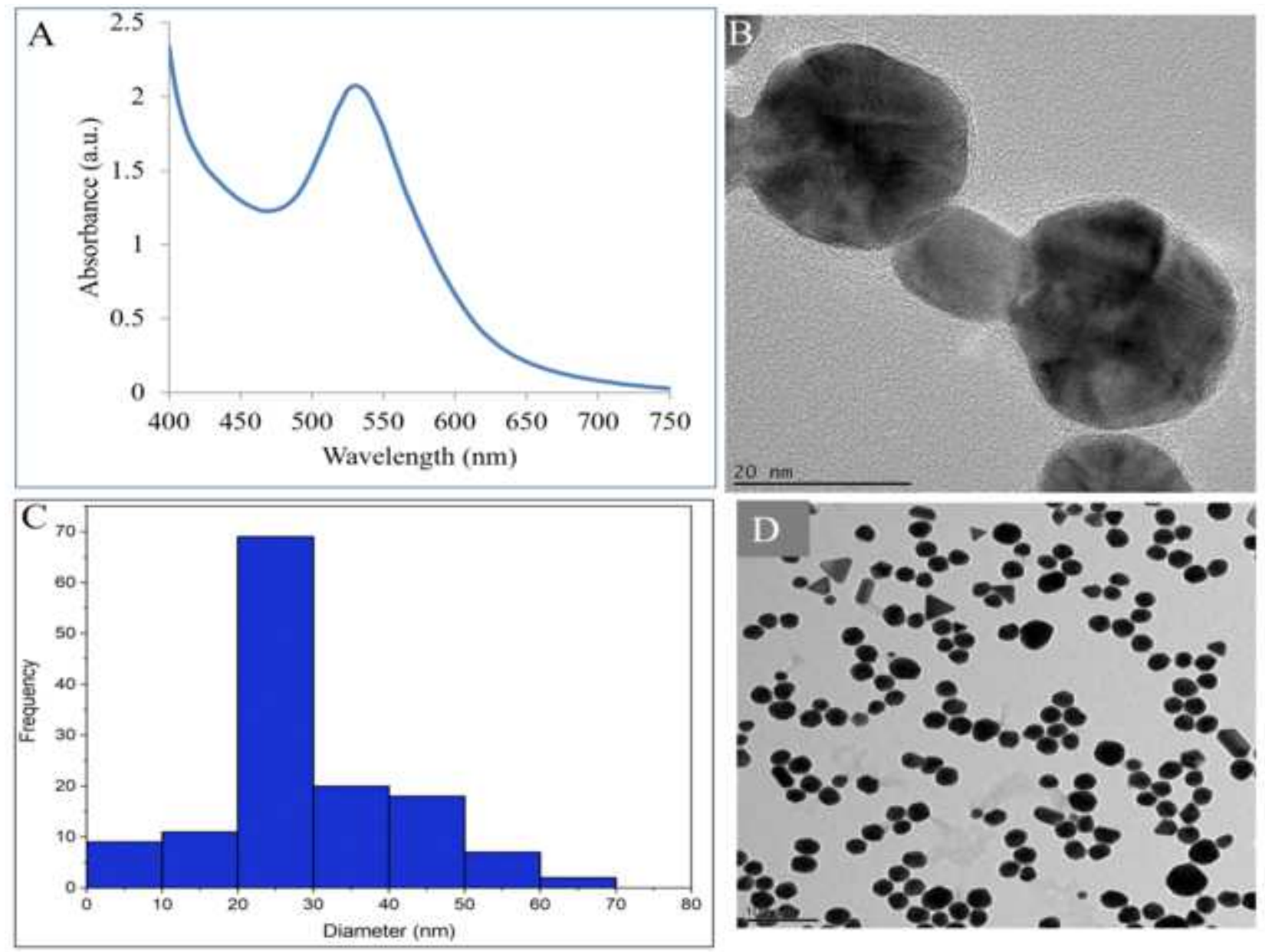

Figure 2

Characterization of MGF-AuNPs by (A) UV-Visible spectra, (B) HR-TEM images, (C) Core size distribution histogram employing image $\mathrm{J}$ software (D) TEM images. Note: TEM: transmission electron microscopy. HR-TEM: high resolution TEM. 


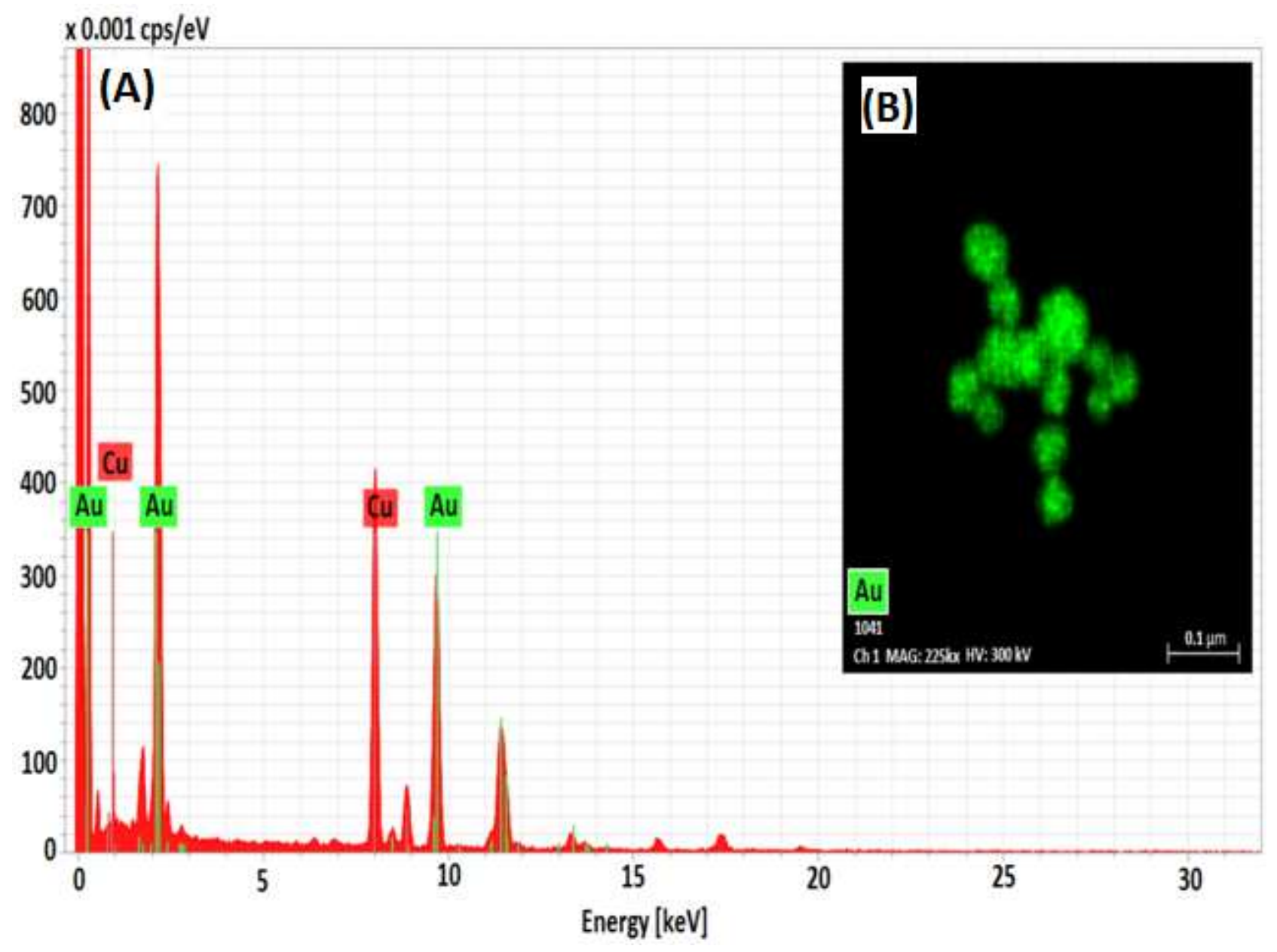

Figure 3

Energy Dispersive Spectroscopy (EDS) analysis of MGF-AuNPs. Image (A) showing the presence of Au element, and image (B) mapping of Au element. 


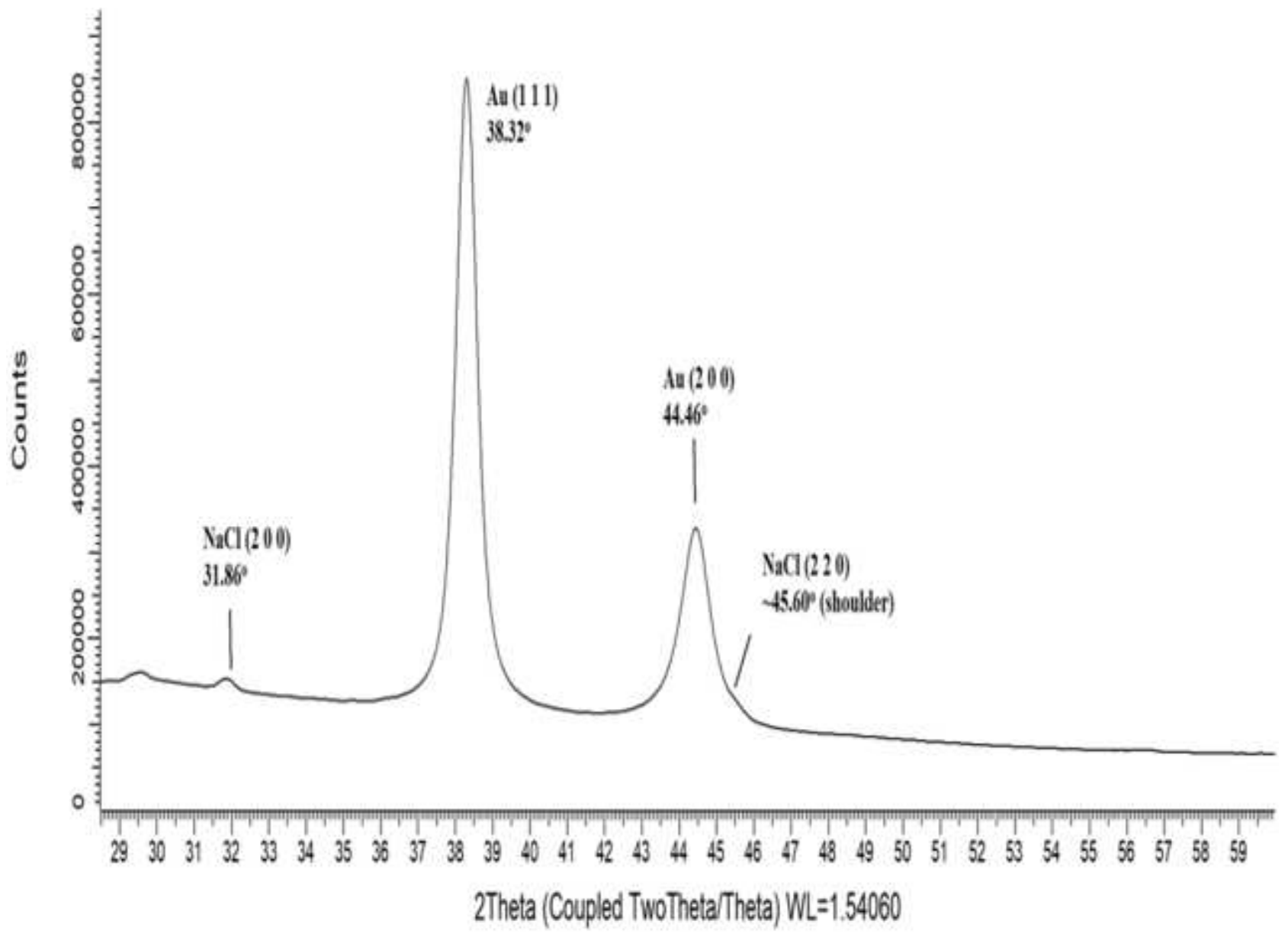

Figure 4

Powder X ray Diffraction (PXRD) pattern of evaporated residue for MGF-AuNPs. 

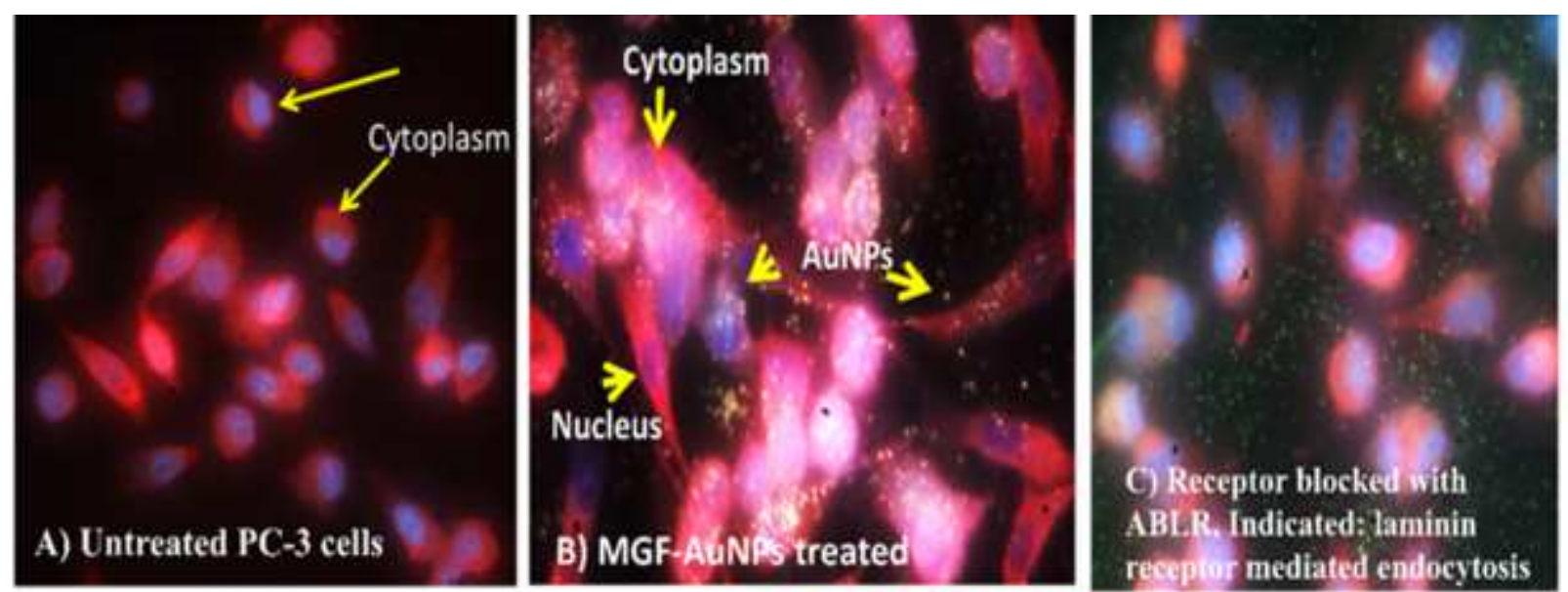

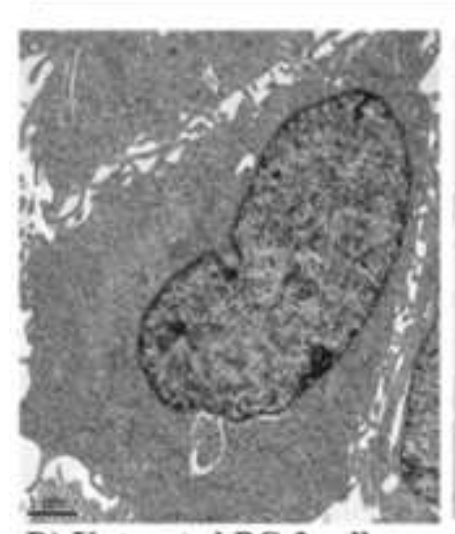

D) Untreated PC-3 cells

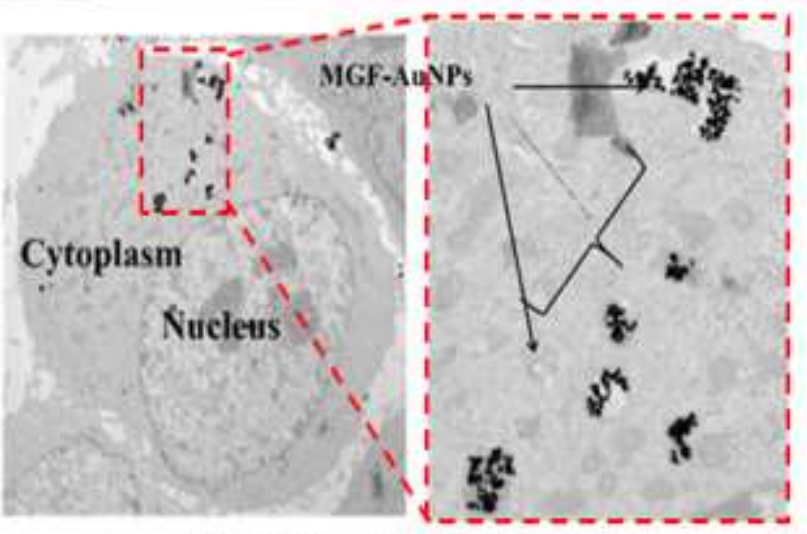

E) MGF-AuNPs treated
F) Receptor blocked with ABLR, Indicated: laminin receptor mediated endocytosis

\section{Figure 5}

Receptor mediated endocytosis of MGF-AuNPs. (A, D): Untreated PC-3 cells; (B, E): MGF-AuNPs (41 $\mu \mathrm{M})$ treated PC-3 cells; (C, F): Laminin receptors on PC-3 cells blocked with ABLR antibody and post treated with MGF-AuNPs, showing laminin receptor affinity of MGF-AuNPs in PC-3 cells. Optical images by cytoviva dark field microscopy and microscopic images by TEM techniques. 


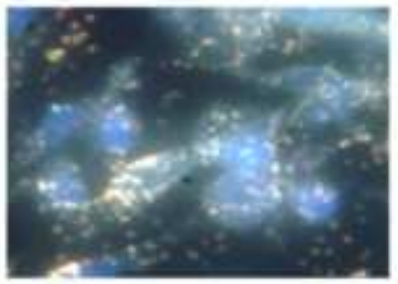

A) MGF-AuNPs treated

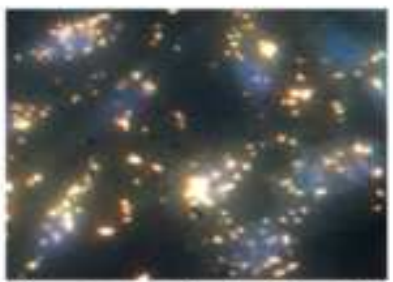

D) Receptor blocked with anti-caveolae AB, Indicated caveolae independent endocytosis

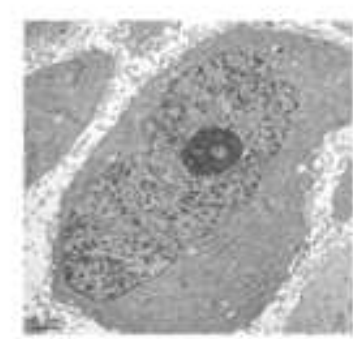

G) Treated with anticlathrin AB; Confirmed clathrin mediated endocytosis

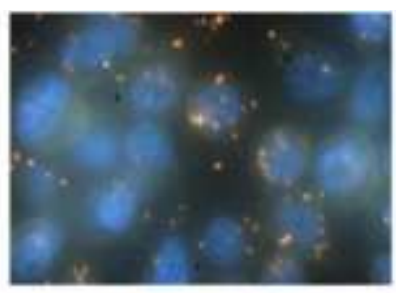

B) Receptor blocked with Chlorpromazine Indicated clathrin mediated endocytosis

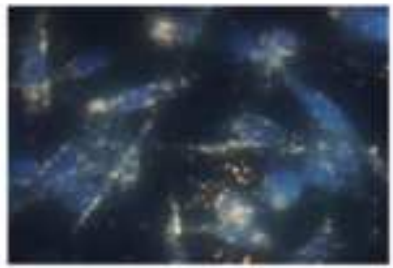

E) Treated with non-specific anti-fibronectin $\mathbf{A B}$ [Fn-3]

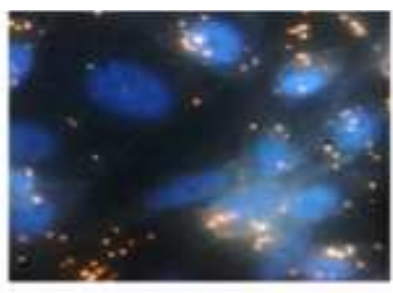

C) Receptor blocked with anticlathrin AB, Indicated clathrin mediated endocytosis

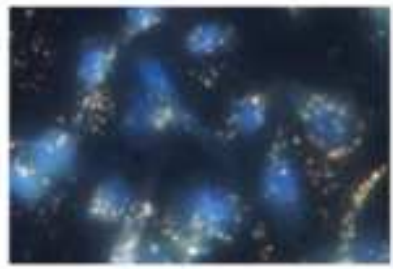

F) Treated with non-specific Mouse IgG Isotype Control

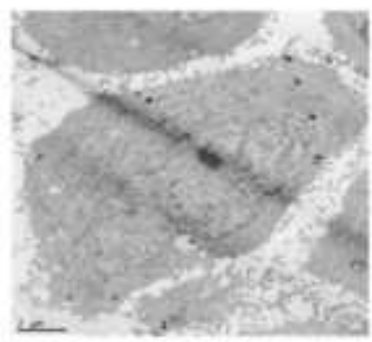

H) Treated with anticaveolae AB; confirmed caveolae independent endocytosis

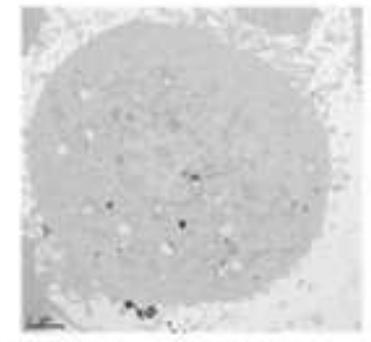

I) Pre-treated with antifibronectin AB [Fn-3], non-specific receptor inhibitor

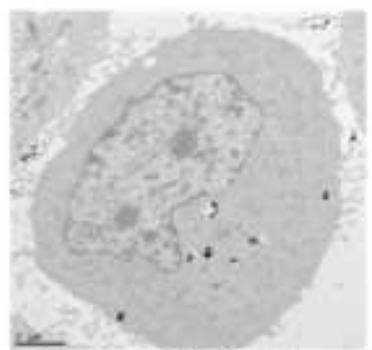

J) Pre-treated with Mouse IgG Isotype Control, nonspecific receptor inhibitor

\section{Figure 6}

Clathrin mediated endocytosis of MGF-AuNPs (A-F): Dark field (CytoViva) microscopic images; (G-J): TEM Images showing PC-3 cells pretreated with Chlorpromazine, anticlarthrin $A B$, anti-caveolae $A B$, antifibronectin $A B$, and mouse IgG Isotype control $A B$, followed by treatment with MGF-AuNPs $(41 \mu M)$. Images infer clathrin dependent and caveolae independent pathways for the endocytosis of MGF-AuNPs in $\mathrm{PC}-3$ cells. 

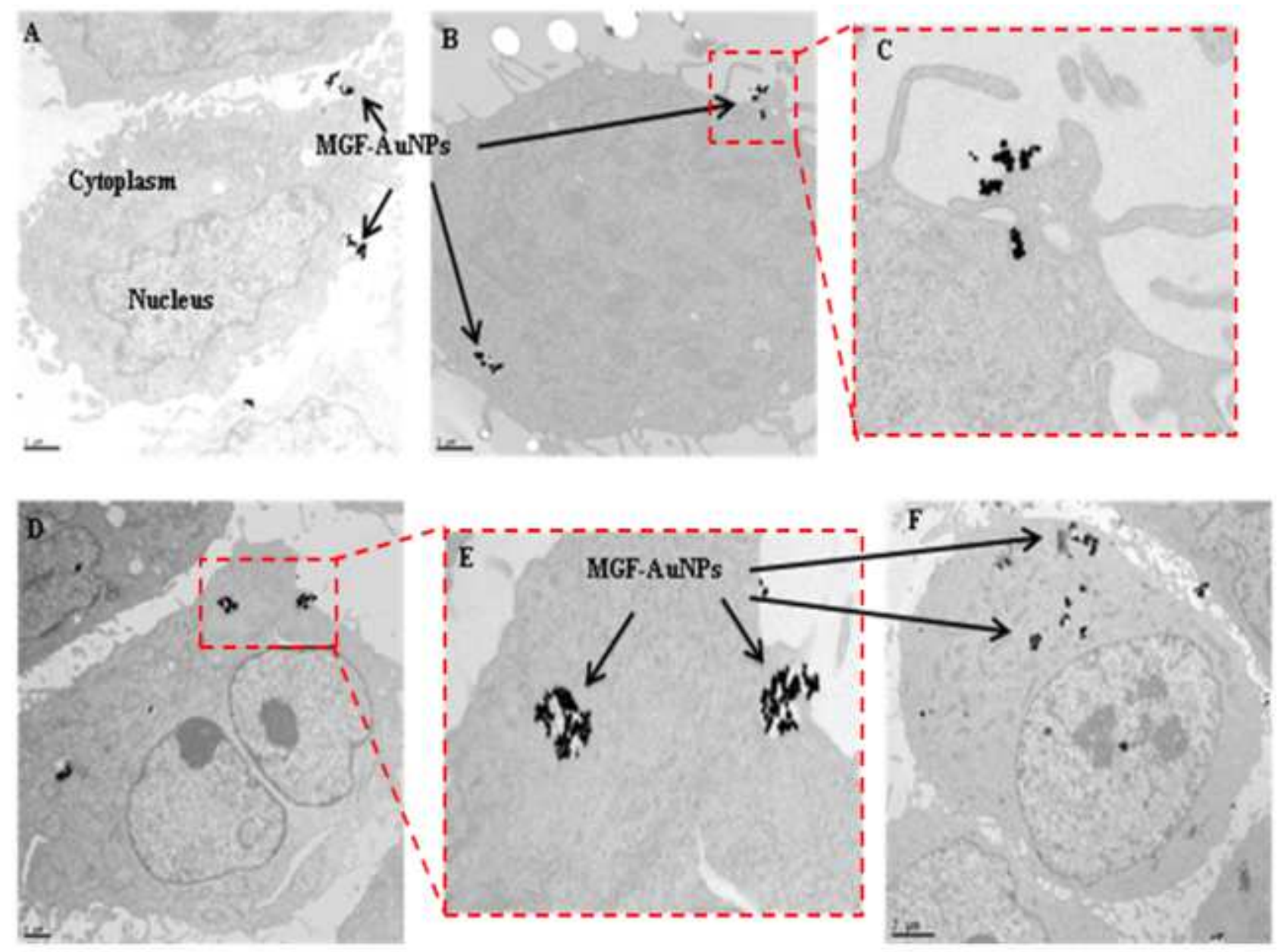

\section{Figure 7}

Time dependent internalization of MGF-AuNPs $(41 \mu \mathrm{M})$ into PC-3 cells, images observed by TEM. (A) at $30 \mathrm{~min}$; (B-C) at $60 \mathrm{~min}$; (D-E) at $90 \mathrm{~min}$; (F) at $120 \mathrm{~min}$. 


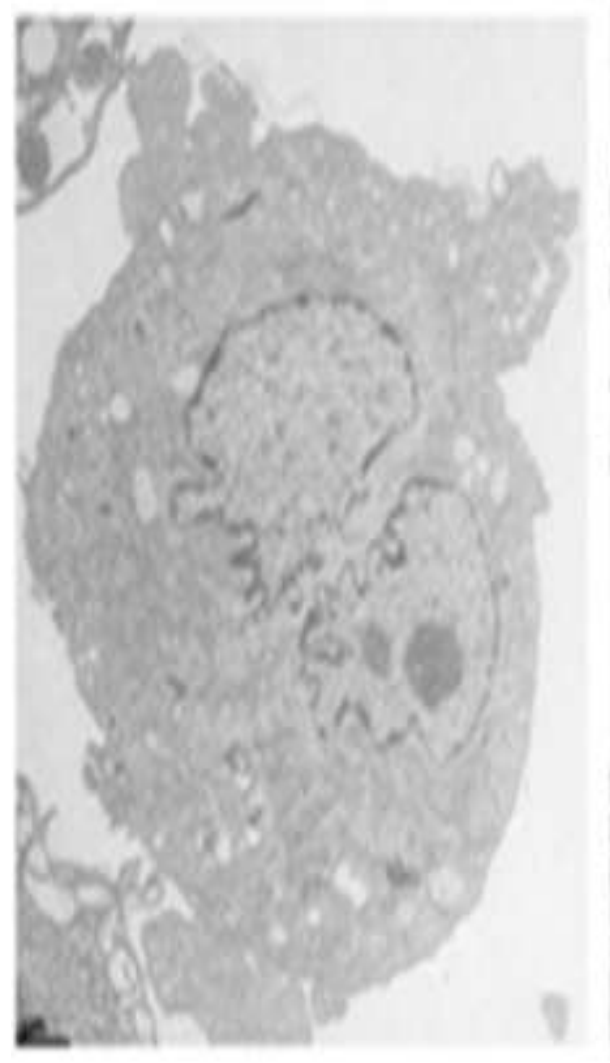

\section{A) Untreated HAECs cells}

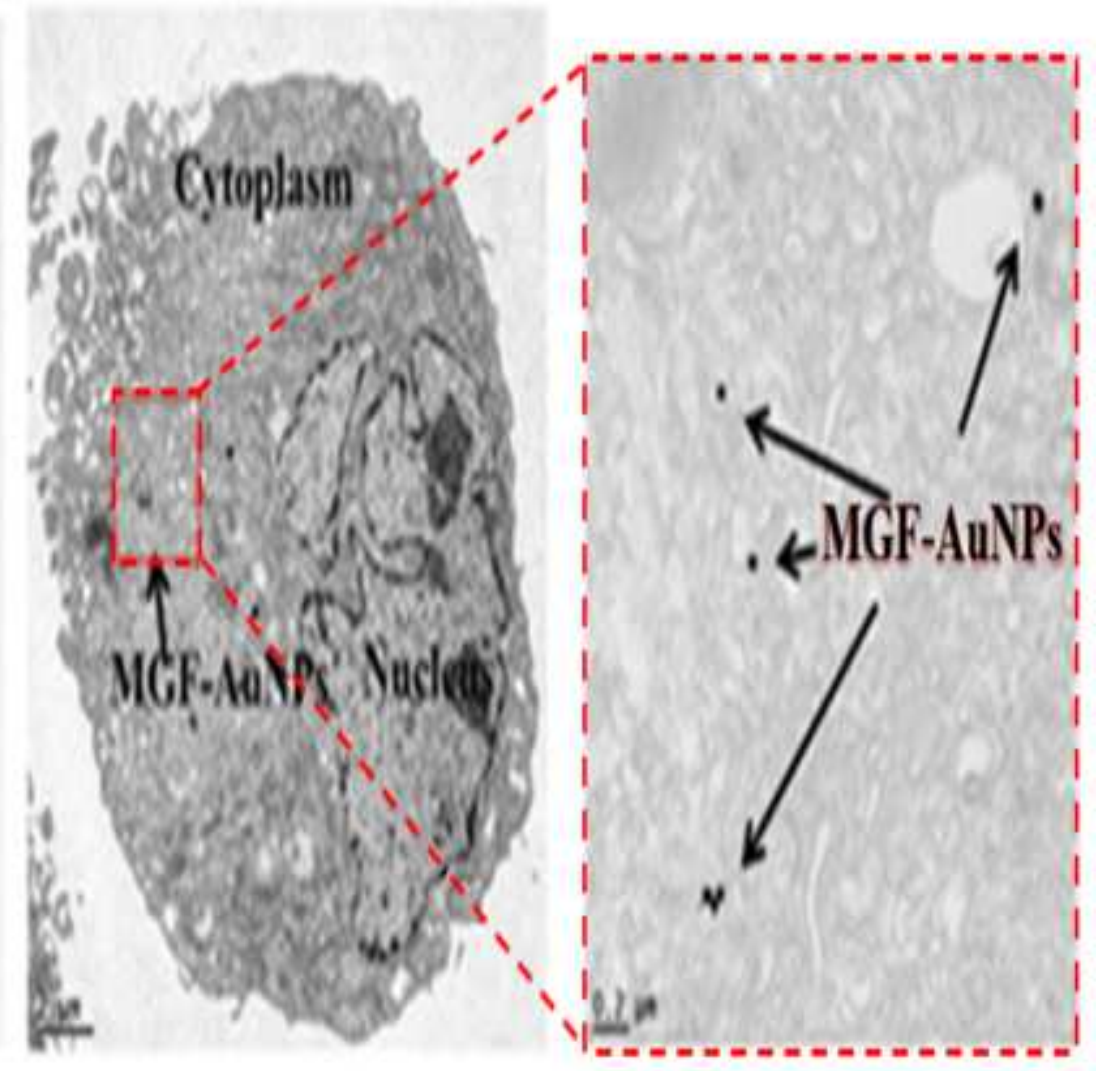

B) MGF-AuNPs treated

Figure 8

TEM images showing MGF-AuNPs uptake into human aortic endothelial cells (HAECs), 60 min post treatment of MGF-AuNPs (41 $\mu \mathrm{M})$. (A) HAECs cells control; no treatment; (B) MGF-AuNPs treated cells (41 $\mu \mathrm{M})$. 


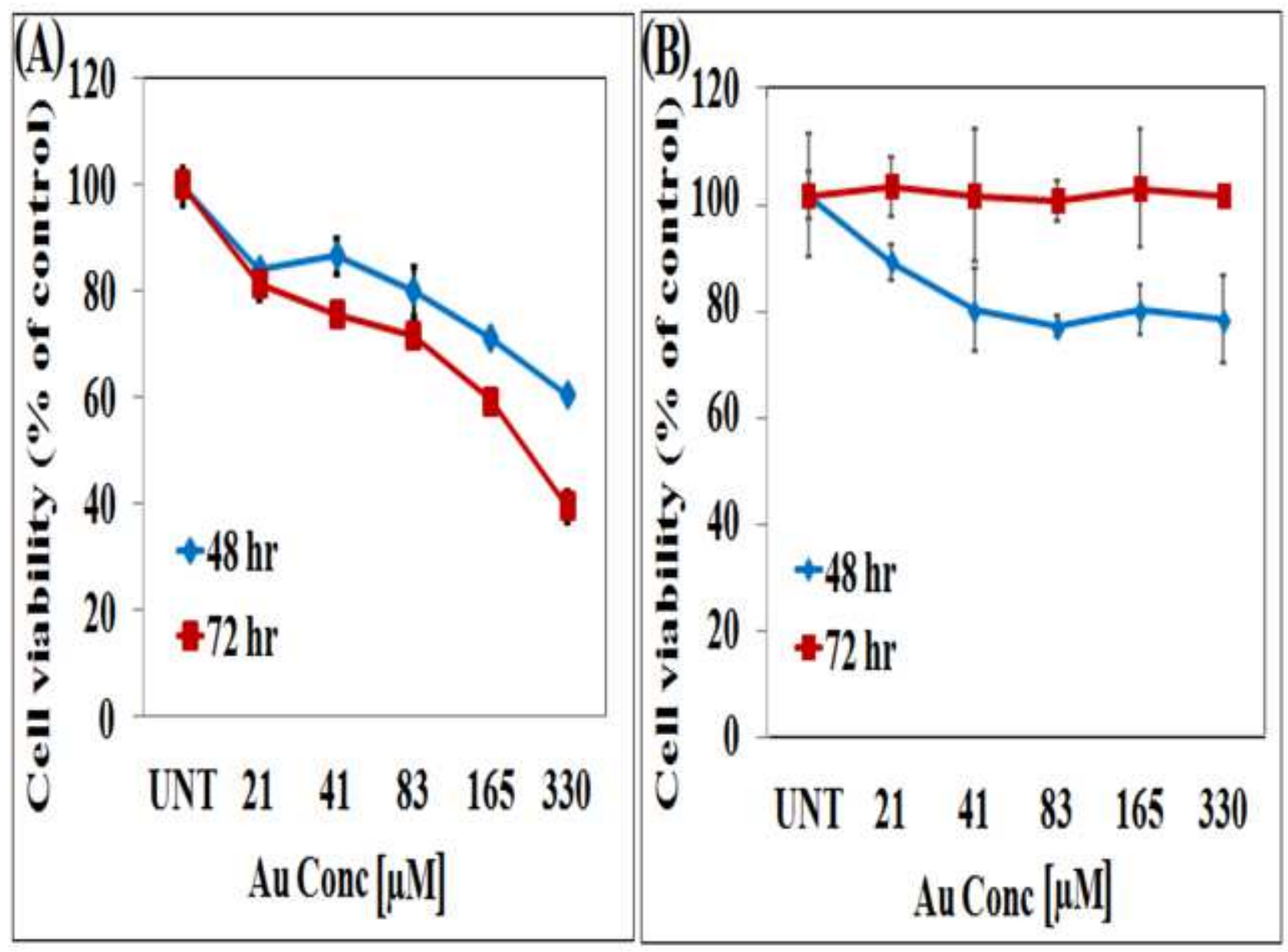

Figure 9

(A) In vitro therapeutic efficacy of MGF-AuNPs against prostate cancer cells (PC-3). (B) MGF-AuNPs showing non-toxic feature to normal cells (HAECs). Cells were treated using serial dilutions of MGFAuNPs and incubated with MTT dye for cell viability measurements. 
A

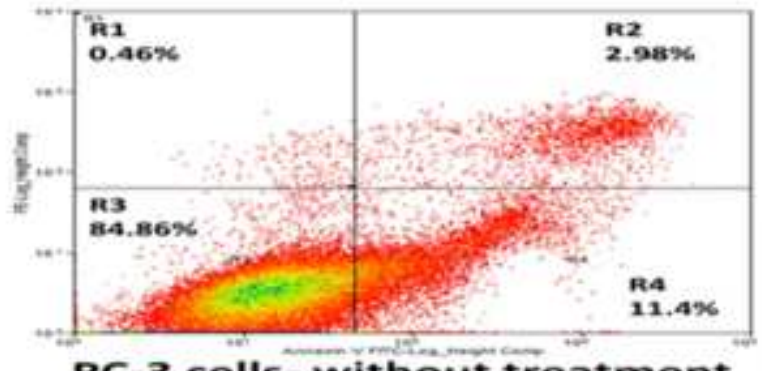

PC-3 cells- without treatment

B

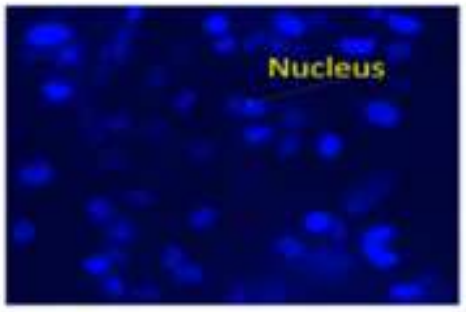

C

PC-3 cells; no treatment
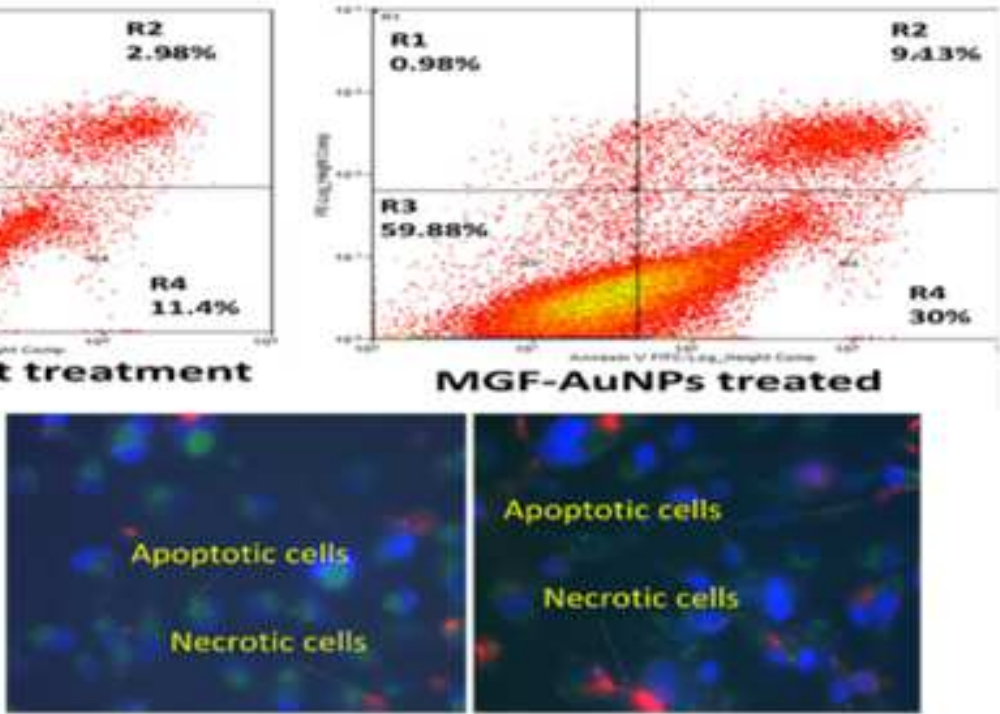

Treated with Std drug

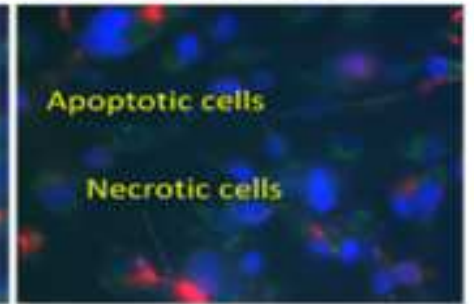

MGFAuNPs treated $83 \mu \mathrm{M}$

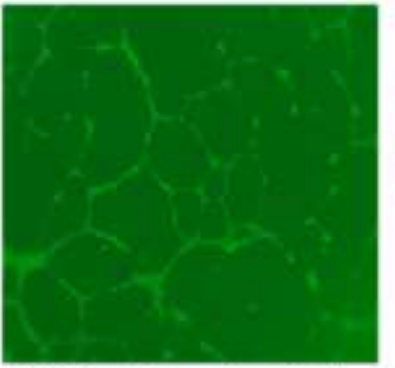

HAEC cells; untreated

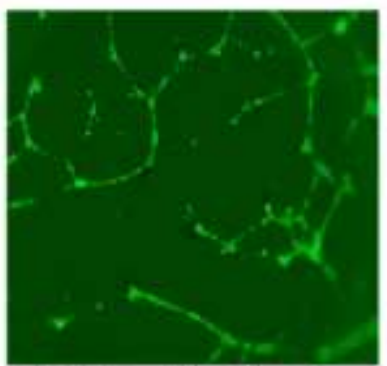

MGF-AuNPs $41 \mu \mathrm{M}$

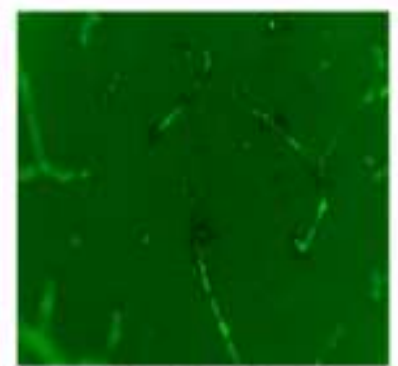

MGF-AuNPs $83 \mu \mathrm{M}$

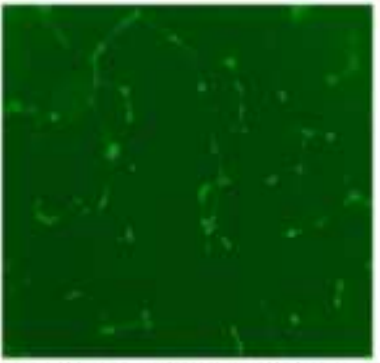

Std drug (Vinblastin) 2 pM

\section{Figure 10}

Apoptosis assays of MGF-AuNPs: (A) induction of apoptosis in PC-3 cells measured by Flow Cytometry; (B) Measured by Fluorescent Microscopy; (C) Inhibition of tube/capillary formation in HAECs endothelial cells confirming anti-angiogenesis effects. 


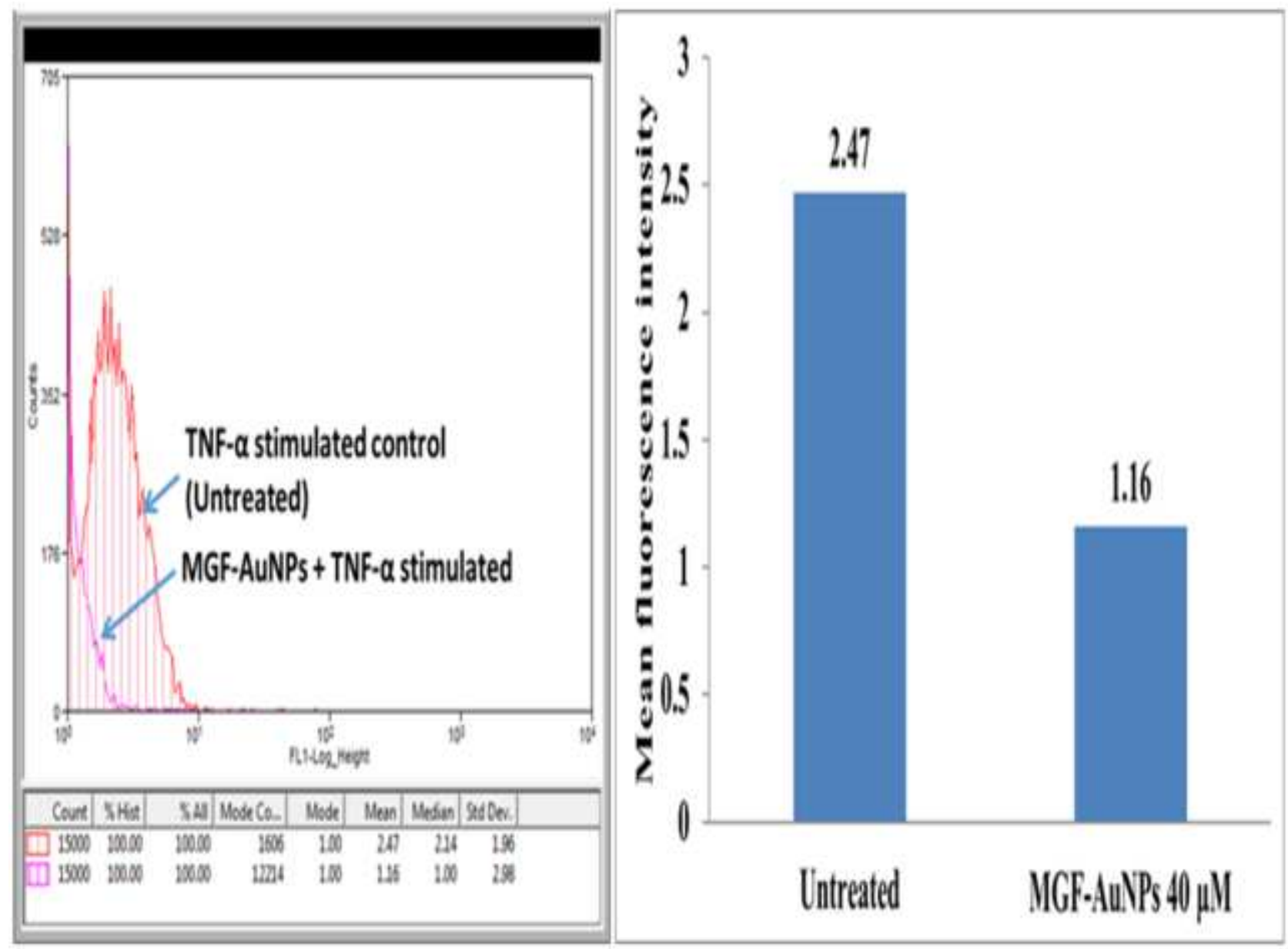

Figure 11

MGF-AuNPs target NF-KB as confirmed through inhibition of TNF-a stimulated NF-kB phosphorylation in PC-3 cells. 


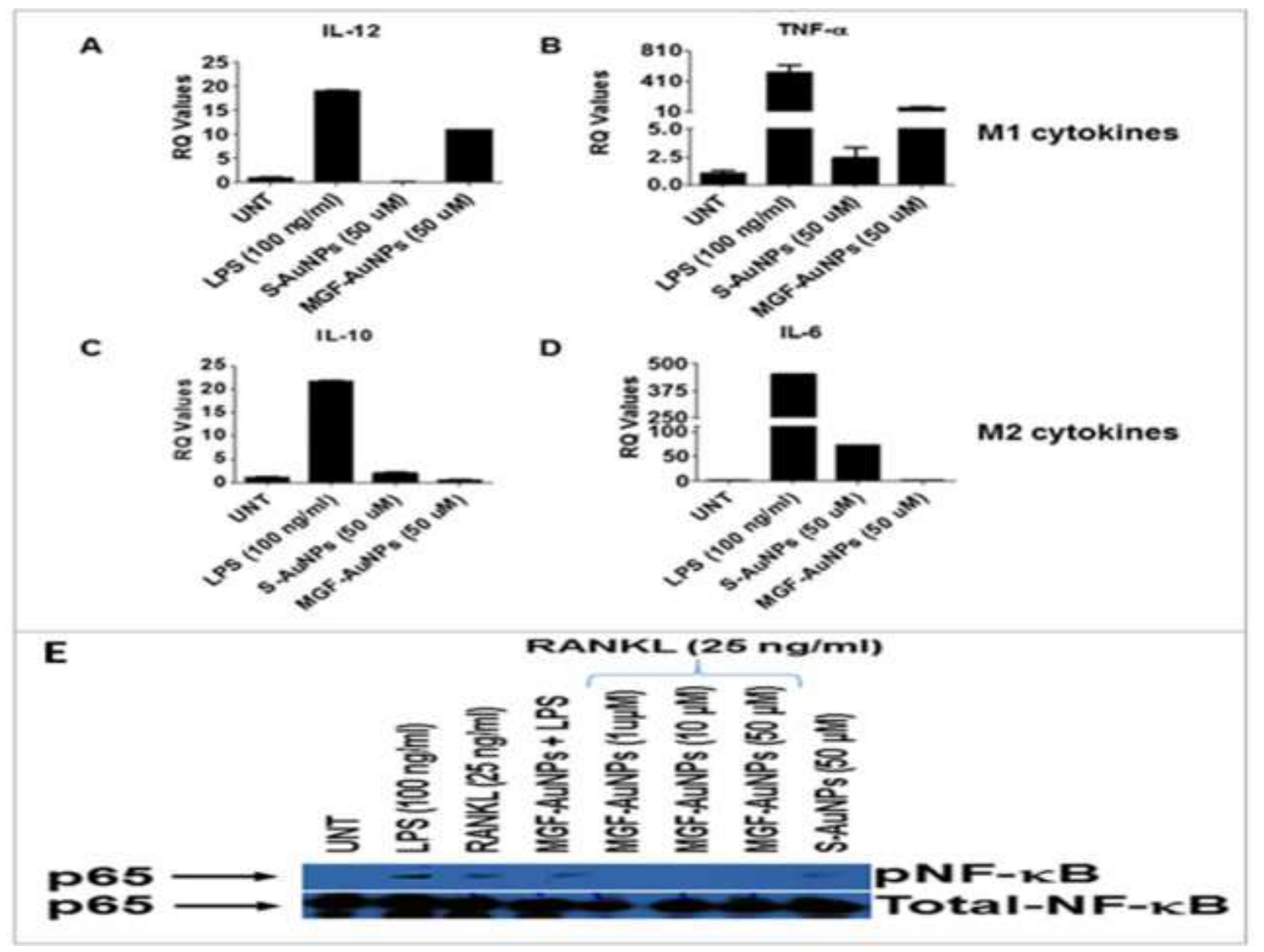

Figure 12

MGF-AuNPs induced polarization of macrophages. (A-D): RAW 264.7 cells were pretreated with either Starch-AuNPs (S-AuNPs as control), or MGF-AuNPs for $2 \mathrm{hr}$ and treated either with LPS $(100 \mathrm{ng} / \mathrm{mL})$ or RANKL $(25 \mathrm{ng} / \mathrm{ml})$ or left untreated for $4 \mathrm{hr}$. RNA was isolated from treated and untreated samples and analyzed for IL-12, TNF-a, IL-10, and IL- 6 by real time PCR using probes from TaqMan, Applied Biosystems. (E): RAW 264.7 cells were either treated with LPS $(100 \mathrm{ng} / \mathrm{mL})$ or Starch-AuNPs (S-AuNPs as control), or MGF-AuNPs or left untreated for 30 mins. The cells were lysed with $1 \mathrm{X}$ Lamellae buffer and lysates were run on PAGE gel and transferred onto nitrocellulose membranes. The membranes were than probed for either phospho- NF-KB or NF-KB using respective antibodies. 


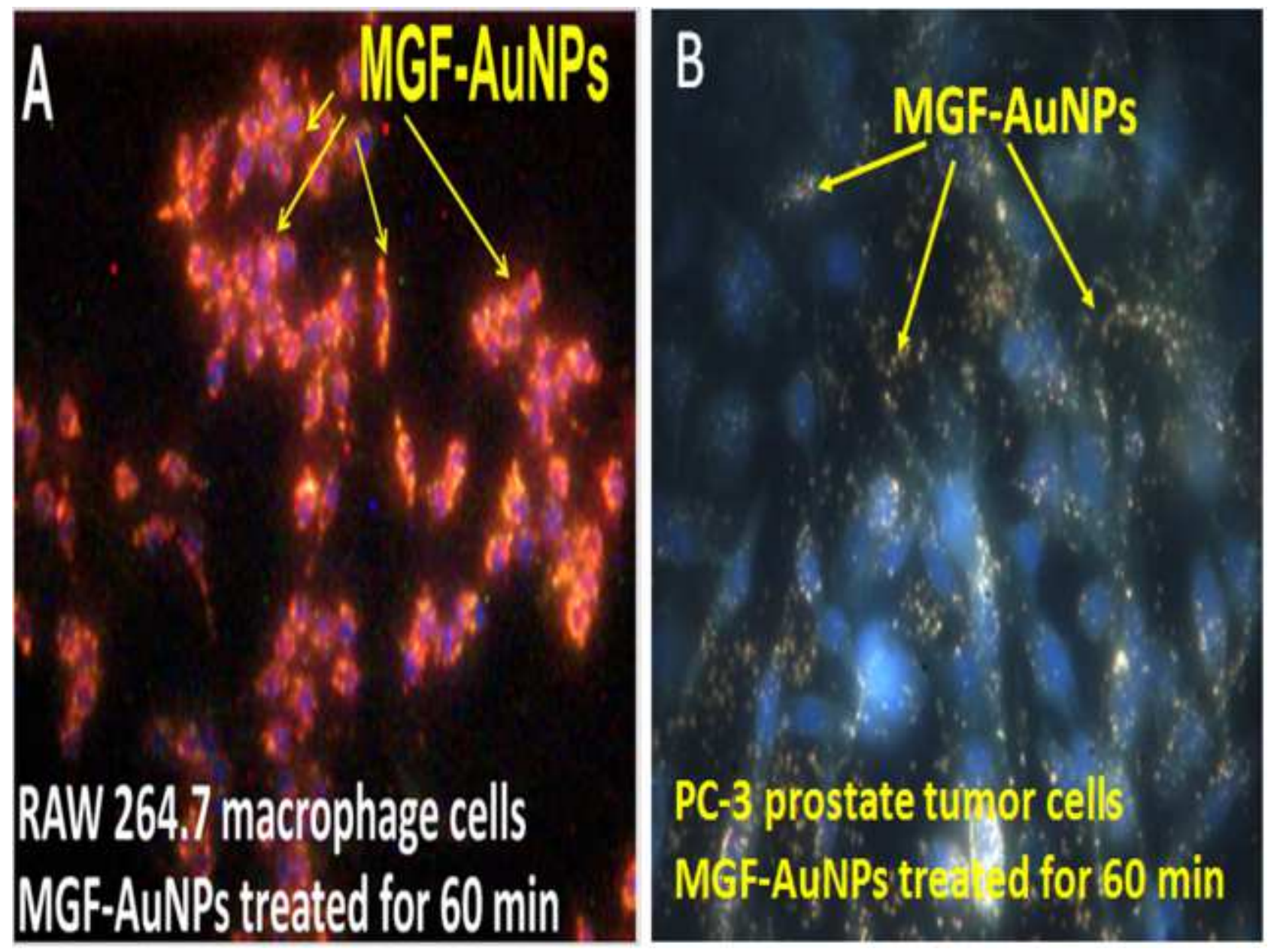

Figure 13

MGF-AuNPs uptake in (A) Raw 264.7 macrophages and (B) PC-3 tumor cells. The cells were post-treated with MGF-AuNPs $(40 \mu \mathrm{M})$ and images were taken using CytoViva dark field microscopy. 


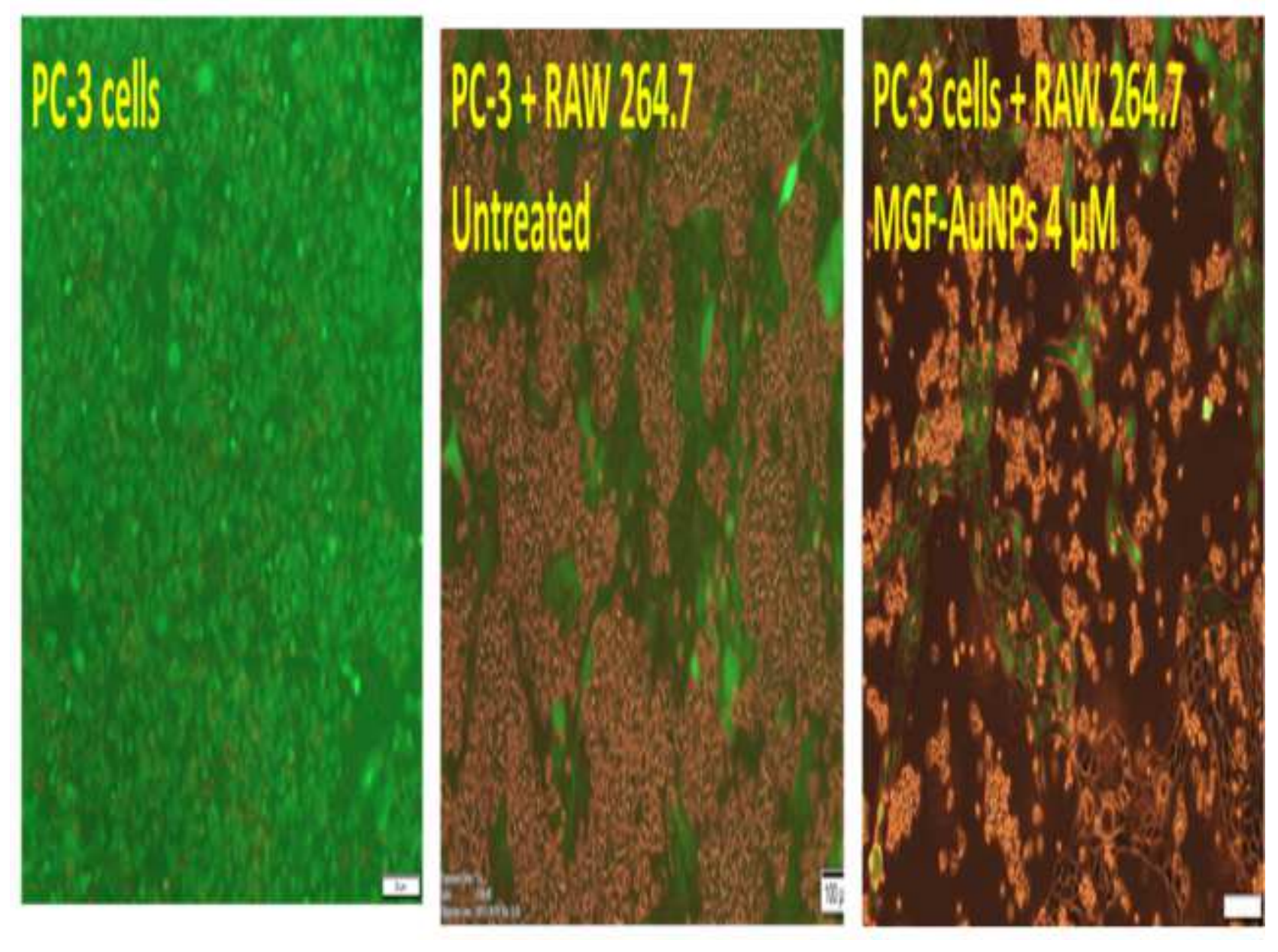

Figure 14

MGF-AuNPs-treated macrophages inhibited the proliferation of prostate tumor cells. Raw 264.7 macrophages were pre-treated with MGF-AuNPs. Separately, PC-3 cells were labelled with CFSE to assess their proliferation. The macrophages were then co-cultured with PC-3 cells for $72 \mathrm{hr}$. The images were obtained by fluorescent microscope. 


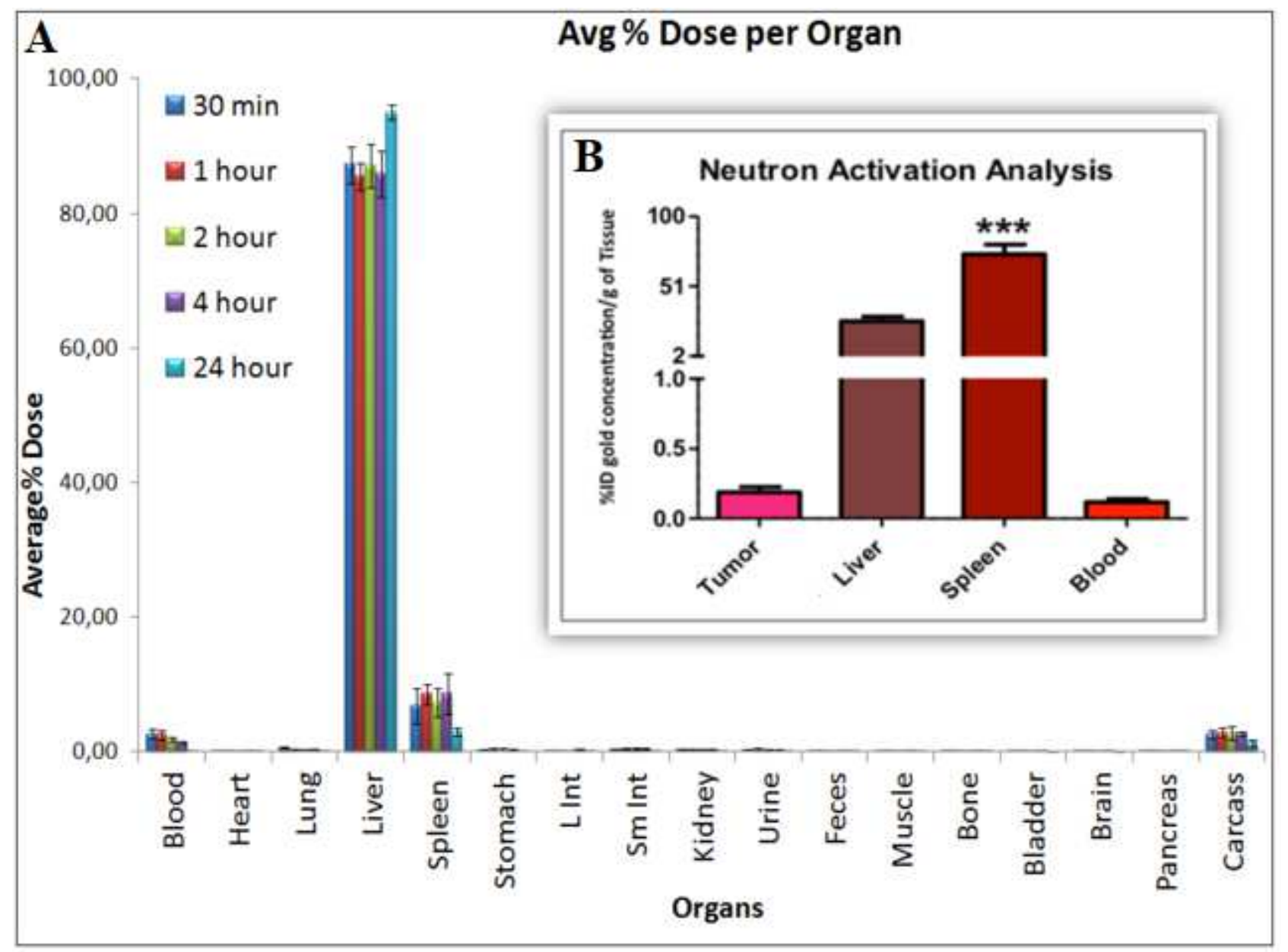

\section{Figure 15}

Targeting of MGF-AuNP on splenic macrophages: (A) Biodistribution of MGF-198AuNP in normal mice showing selective uptake only in liver and spleen and limited/no uptake in non-target organs; (B) Biodistribution of MGF-198AuNPs in prostate tumor bearing SCID mice. Gold concentrations in tumor, liver and spleen measured using neutron activation analysis (NAA) - showing limited uptake in tumors and major uptake in spleen and liver-suggesting targeting of MGF-AuNP on splenic macrophages. 


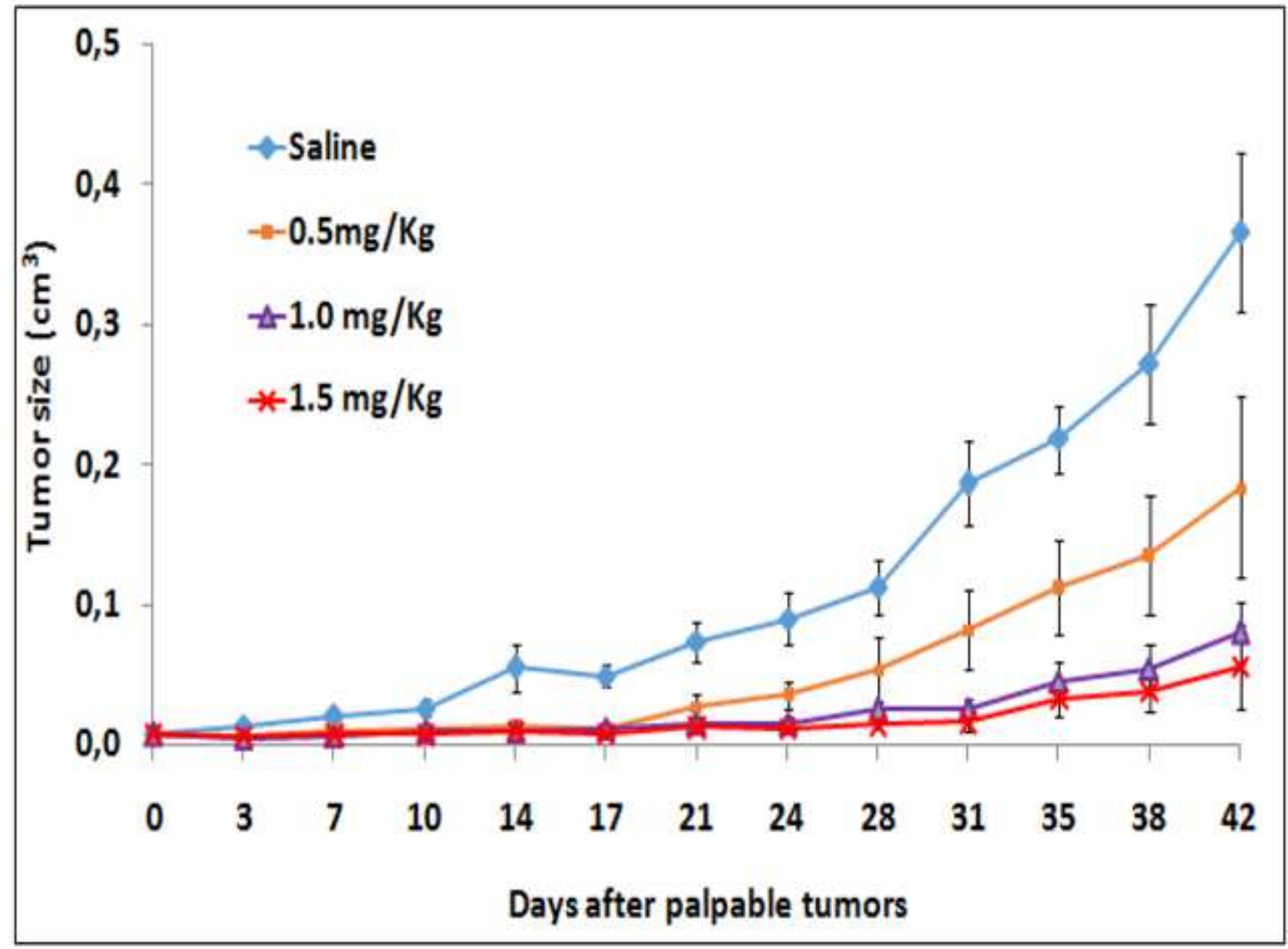

Figure 16

In vivo therapeutic efficacy of MGF-AuNPs in SCID mice implanted with PC-3 prostate tumor cells ( $n=7 /$ set). MGF-AuNPs were administered intraperitoneally.

\section{Supplementary Files}

This is a list of supplementary files associated with this preprint. Click to download.

- Table2ArriveDocumentKhoobchandanietalScientificReports.pdf

- Figures1.tiff

- SupplementarysectionMGFAuNPspaper.pdf

- Scheme1.png

- Scheme3.png 